\title{
Quantum effects near the Cauchy horizon of a Reissner-Nordström black hole
}

\author{
Orr Sela \\ Department of physics, Technion-Israel Institute of Technology, Haifa 32000, Israel
}

\begin{abstract}
We consider a massless, minimally-coupled quantum scalar field on a Reissner-Nordström black hole background, and we study the leading asymptotic behavior of the expectation value of the stress energy tensor operator $\left\langle\hat{T}_{\mu \nu}\right\rangle_{\text {ren }}$ and of $\left\langle\hat{\Phi}^{2}\right\rangle_{\text {ren }}$ near the inner horizon, in both the Unruh and the Hartle-Hawking quantum states. We find that the coefficients of the expected leading-order divergences of these expectation values vanish, indicating that the modifications of the classical geometry due to quantum vacuum effects might be weaker than expected. In addition, we calculate the leading-order divergences of $\left\langle\hat{T}_{\mu \nu}\right\rangle_{\text {ren }}$ and of $\left\langle\hat{\Phi}^{2}\right\rangle_{\text {ren }}$ in the Boulware state near the outer (event) horizon, and we obtain analytical expressions that correspond to previous numerical results.
\end{abstract}

\section{INTRODUCTION}

The classical Einstein's field equation of general relativity admits black hole (BH) solutions with internal structures that possess exotic features such as naked singularities, Cauchy horizons and bridges to other universes. However, this is an ideal picture obtained when considering a highly symmetric, isolated black hole. Two well known solutions that have this kind of nontrivial internal structure are the Reissner-Nordström (RN) solution, describing a spherically symmetric, electrically charged $\mathrm{BH}$, and the Kerr solution, describing a stationary, rotating and axially-symmetric $\mathrm{BH}$.

As it turns out, when one adds (classical) external matter and perturbations to the picture, the internal region of these BH solutions is highly modified and a null curvature singularity is developed in the ingoing section of the inner horizon - the Cauchy horizon. This singularity has a very interesting nature - it is a weak singularity, meaning that the metric there is continuous but not differentiable. As a result, an observer can pass through this singularity and experience a regular physical effect (such as finite tidal forces). This phenomenon has been shown to occur for both (four dimensional) spherical charged black holes [1-3] and rotating black holes [4 -7]. Moreover, this picture is qualitatively the same both for a test-field analysis and for an analysis that takes into account the back-reaction of the perturbations. Recently, it was shown that even on the outgoing section of the inner horizon an interesting singularity is developed - a shock-wave singularity [8, 9].

In addition to these classical effects, a very important source for stress-energy (and as a result for back-reaction) is the quantum nature of fields. As Hawking [10, 11] showed, considering quantum fields on a (classical) BH background might result in a significant effect on the geometry (at least if taken for long enough time) and change one's qualitative picture of this spacetime. In particular, he showed that a BH should evaporate through an emission of radiation, and therefore the entire structure of this $\mathrm{BH}$ spacetime turns out to be very different than the one proposed by the classical picture.

In this paper, we aim at investigating the stress-energy resulting from quantum fields in the interior region of a BH with a nontrivial internal structure (as discussed above). Specifically, we are interested in the behavior of the stress-energy tensor near the Cauchy horizon, where it is expected to diverge [12 14]. Analyzing this divergence can provide us an important insight into the possible modification of the interior geometry caused by the quantum theory.

We consider the framework of semiclassical general relativity, in which the gravitational field is treated classically as a curvature of spacetime, while all the other fields are taken as quantum fields residing in this background. Therefore, instead of the classical Einstein's field equation, we now have the following:

$$
G_{\mu \nu}=8 \pi\left\langle\hat{T}_{\mu \nu}\right\rangle_{r e n}
$$

where $G_{\mu \nu}$ is the Einstein tensor of the background geometry and $\left\langle\hat{T}_{\mu \nu}\right\rangle_{r e n}$ is the renormalized expectation value of the stress-energy tensor operator associated with the quantum fields. In Eq. (1.1) and throughout this paper we adopt standard geometric units $c=G=1$ and the signature $(-+++)$.

In Ref. [12, it was shown that the renormalized stress-energy tensor (RSET), calculated in the Hartle-Hawking state on a two dimensional RN black hole background, diverges at the inner horizon. Then, it was claimed that also in four dimensions the RSET is expected to diverge at the inner horizon. However, this divergence was only claimed on general grounds, without any actual calculation.

In Refs. [13, 14, it was shown that the RSET, calculated in the Unruh state on a four dimensional RN black hole background, must diverge on at least one of the two inner horizons. It was also shown that this result applies to slowly rotating black holes as well under the assumption that some components of the RSET are continuous functions 
of $a$, the Kerr angular momentum parameter. A stronger assumption, that of analyticity in $a$, yields this divergence for a general value of $a$. This divergence, in turn, suggests that the classical picture is strongly modified by quantum vacuum effects associated with the Hawking evaporation process. However, as in [12], the form of this divergence was not found.

Our goal in this work is to analyze these expected divergences at the inner horizons of black holes with a nontrivial internal structure. For this purpose, we consider a simple model with the above mentioned features: A massless, minimally coupled quantum scalar field on a RN black hole background. Being massless and minimally coupled, the scalar field operator $\hat{\Phi}(x)$ satisfies the d'alembertian equation:

$$
\square \hat{\Phi}(x)=0,
$$

where the metric used in the calculation of this d'alembertian is the RN metric. Then, after finding the asymptotic form (near the inner horizon) of the scalar field modes involved in the expression for the RSET, we calculate the expected leading-order divergence of this RSET near the inner horizon and show that its coefficient vanishes. This, in turn, suggests that the modification of the geometry might be weaker than expected. Our logic is based on experience from investigating two dimensional black hole models, where the asymptotic form of the RSET near the inner horizon determines the modification of the geometry there, even when back-reaction is taken into account [15].

An important point in our calculation of the asymptotic form of the modes near the inner horizon is that the large- $l$ limit is taken, where $l$ is the usual number appearing in the angular decomposition of the modes into spherical harmonics. That is, we assume that the leading-order behavior of $\left\langle\hat{T}_{\mu \nu}\right\rangle_{r e n}$ and $\left\langle\hat{\Phi}^{2}\right\rangle_{\text {ren }}$ near the inner horizon is determined by the large- $l$ modes. In the external region of the $\mathrm{BH}$, this assumption turns out to yield the exact asymptotic form of the RSET near the outer horizon numerically computed in [16, 17], and in the internal region it is consistent with the numerical results of [18] that will be published elsewhere (see below for more details). For other approximation schemes which have been employed in different cases than the one considered in this paper, see [19] for an approximation of the RSET of a conformally coupled scalar field on a RN background, and 20 23. for related discussions. See also [16, 24].

The organization of this paper is as follows. We start in section II with the preliminaries needed for our analysis. Then, in section III, and before we turn to the analysis in the interior region of the BH, we apply our analytical approach to the calculation of the leading-order divergence of the RSET (and of $\left\langle\hat{\Phi}^{2}\right\rangle_{\text {ren }}$, where $\hat{\Phi}$ is the scalar field operator) in Boulware state upon approaching the outer (event) horizon from outside of the BH. Later, in section IV, we review some useful results from [25], where the two-point function in the interior region was expressed in terms of a radial function (or alternatively, in terms of some inner modes) that can be analytically calculated in the asymptotic region near the inner horizon. Then, in section $\mathrm{V}$, we find this asymptotic form of the radial function and use it to calculate in section VI the leading divergence of $\left\langle\hat{\Phi}^{2}\right\rangle_{r e n}$ and $\left\langle\hat{T}_{\mu \nu}\right\rangle_{r e n}$ near the inner horizon. We finally conclude in section VII.

\section{PRELIMINARIES}

\section{A. Coordinate systems and quantum states}

In this paper we consider the Reissner-Nordström spacetime, which in the standard Schwarzschild coordinates has the following metric:

$$
d s^{2}=-\left(1-\frac{2 M}{r}+\frac{Q^{2}}{r^{2}}\right) d t^{2}+\left(1-\frac{2 M}{r}+\frac{Q^{2}}{r^{2}}\right)^{-1} d r^{2}+r^{2}\left(d \theta^{2}+\sin ^{2} \theta d \varphi^{2}\right) .
$$

We define the various choices of coordinates on this space following [25]. First, we define the tortoise coordinate, $r_{*}$, using the standard relation

$$
\frac{d r}{d r_{*}}=1-\frac{2 M}{r}+\frac{Q^{2}}{r^{2}}
$$

We use this relation to define $r_{*}$ both in the interior and the exterior regions of the BH. More explicitly, we choose the integration constants such that $r_{*}$ is given by

$$
r_{*}=r+\frac{1}{2 \kappa_{+}} \ln \left(\frac{\left|r-r_{+}\right|}{r_{+}-r_{-}}\right)-\frac{1}{2 \kappa_{-}} \ln \left(\frac{\left|r-r_{-}\right|}{r_{+}-r_{-}}\right)
$$




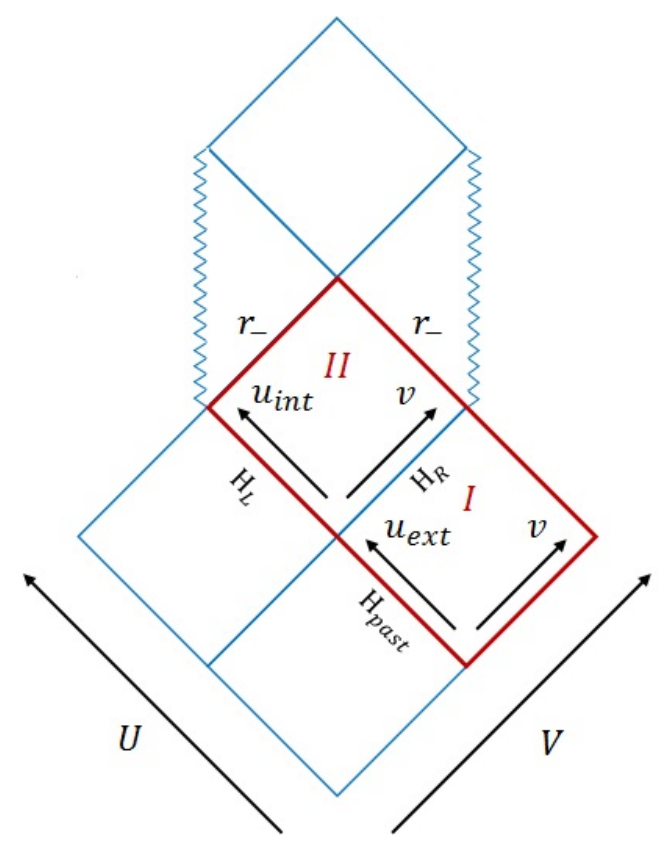

Figure 1: Penrose diagram of Reissner-Nordström spacetime. In the exterior region, region $I$ in the figure, we use the exterior Eddington-Finkelstein coordinates, while in the interior, region $I I$ in the figure, we use the interior Eddington-Finkelstein coordinates. In addition, the Kruskal coordinate system is shown and is defined in both regions $I$ and $I I$. The red-framed area denotes the region in the eternal Reissner-Nordström spacetime which concerns this paper, i.e. regions $I$ and $I I$.

in both regions, where $\kappa_{ \pm}$are the surface gravities of the $\mathrm{BH}$ corresponding to the inner and outer horizons, and are defined as

$$
\kappa_{ \pm}=\frac{r_{+}-r_{-}}{2 r_{ \pm}^{2}}
$$

Notice that both $\kappa_{+}$and $\kappa_{-}$are chosen to be positive. Using Eq. 2.1), it is easy to see that the outer horizon (at $r=r_{+}$) corresponds to $r_{*} \rightarrow-\infty$ (both for $r_{*}$ defined in the exterior region and for that defined in the interior) and the inner horizon (at $r=r_{-}$) to $r_{*} \rightarrow \infty$.

Next, we define the Eddington-Finkelstein coordinates in the exterior region by

$$
u_{\mathrm{ext}}=t-r_{*} \quad, \quad v=t+r_{*},
$$

while in the interior region by

$$
u_{\text {int }}=r_{*}-t \quad, \quad v=r_{*}+t,
$$

see Fig. (1). The Kruskal coordinates corresponding to the event horizon, $r=r_{+}$, are defined in terms of the exterior and interior Eddington-Finkelstein coordinates by

$$
U\left(u_{\mathrm{ext}}\right)=-\frac{1}{\kappa_{+}} \exp \left(-\kappa_{+} u_{\mathrm{ext}}\right), \quad U\left(u_{\mathrm{int}}\right)=\frac{1}{\kappa_{+}} \exp \left(\kappa_{+} u_{\mathrm{int}}\right), \quad V(v)=\frac{1}{\kappa_{+}} \exp \left(\kappa_{+} v\right) .
$$

Note that we are interested in regions I and II of Fig. (1) [i.e. the region $(-\infty<U<\infty, V>0)$ ], in which the coordinate $v$ is well defined and so we do not need to introduce different coordinates, $v_{\text {ext }}$ and $v_{\text {int }}$, for the exterior and interior regions.

We make the following notations: $H_{\text {past }}$ denotes the past horizon [i.e. the region $(U<0, V=0)$ ], PNI denotes past-null-infinity [i.e. $(U=-\infty, V>0)$ ], $H_{L}$ is the region $(U>0, V=0)$ and $H_{R}$ is the region $(U=0, V>0)$, see Fig. (1). We call $H_{L}$ and $H_{R}$ the "left event horizon" and "right event horizon", respectively.

Let us now define the three quantum states that we consider in this paper. Again, we follow the notations of [25]. We begin with defining the Boulware state [26] which is defined in the exterior region of the BH. In order to do so, we decompose our (massless, minimally coupled) scalar field operator $\hat{\Phi}(x)$ in the exterior region using two independent 
sets of modes $f_{\omega l m}^{\Lambda}(x)$, known as the Boulware modes, where $\Lambda$ denotes "in" and "up". The decomposition is given by

$$
\hat{\Phi}(x)=\int d \omega \sum_{\Lambda, l, m}\left[f_{\omega l m}^{\Lambda}(x) \hat{a}_{\omega l m}^{\Lambda}+f_{\omega l m}^{\Lambda *}(x) \hat{a}_{\omega l m}^{\Lambda \dagger}\right]
$$

and the Boulware modes $f_{\omega l m}^{\Lambda}$ are defined as follows. First, these modes are solutions of the d'alembertian equation satisfied by the scalar field operator $\hat{\Phi}(x)$ [see Eq. [1.2] ], i.e.

$$
\square f_{\omega l m}^{\Lambda}(x)=0 .
$$

Exploiting the spherical symmetry, we can decompose $f_{\omega l m}^{\Lambda}$ as

$$
f_{\omega l m}^{\Lambda}(x)=|\omega|^{-1 / 2} C_{l m}(x) \tilde{f}_{\omega l}^{\Lambda}(x)
$$

where

$$
C_{l m}(x)=(4 \pi)^{-1 / 2} \frac{1}{r} Y_{l m}(\theta, \varphi)
$$

and get a two-dimensional wave equation for $\tilde{f}_{\omega l}^{\Lambda}$ :

$$
\tilde{f}_{, r_{*} r_{*}}^{\Lambda}-\tilde{f}_{, t t}^{\Lambda}=V_{l}(r) \tilde{f}^{\Lambda}
$$

where

$$
V_{l}(r)=\left(1-\frac{2 M}{r}+\frac{Q^{2}}{r^{2}}\right)\left[\frac{l(l+1)}{r^{2}}+\frac{2 M}{r^{3}}-\frac{2 Q^{2}}{r^{4}}\right]
$$

The Boulware modes are then defined by demanding that $\tilde{f}_{\omega l}^{\Lambda}$ are the solutions of Eq. 2.7) that satisfy the following initial conditions:

$$
\begin{gathered}
\tilde{f}_{\omega l}^{\text {in }}=\left\{\begin{array}{cc}
0, & H_{\text {past }} \\
e^{-i \omega v}, & \text { PNI }
\end{array},\right. \\
\tilde{f}_{\omega l}^{\text {up }}=\left\{\begin{array}{cc}
e^{-i \omega u_{\text {ext }}}, & H_{\text {past }} \\
0, & \text { PNI }
\end{array}\right.
\end{gathered}
$$

The Boulware state, $|0\rangle_{B}$, is then defined by

$$
\hat{a}_{\omega l m}^{\Lambda}|0\rangle_{B}=0
$$

Note that these modes and quantum state are defined in the exterior region of the BH [region I of Fig. (1)]. This state corresponds to the familiar concept of an empty state at spatial infinity, in the sense that the expectation value of the stress-energy tensor (in asymptotically Lorentzian coordinate system) in this state goes to zero at large radii [27. Moreover, this expectation value, evaluated in a freely falling frame, diverges at the event horizons.

An alternative and natural name for the Boulware modes that we will use in this paper is the "outer EddingtonFinkelstein modes". Analogously to the definition of these modes, we can also define "inner Eddington-Finkelstein modes". We shall use a similar notation for these modes, $f_{\omega l m}^{\Lambda}$ and $\tilde{f}_{\omega l}^{\Lambda}$ [related by Eq. [2.5]], but with $\Lambda$ denoting "right" $(R)$ and "left" $(L)$ instead of "in" and "up". These modes are defined in the interior region of the BH [region II of Fig. [1] by the following initial conditions on the left and right event horizons:

$$
\begin{aligned}
& \tilde{f}_{\omega l}^{\mathrm{L}}=\left\{\begin{array}{cc}
e^{-i \omega u_{\mathrm{int}}} & , H_{L} \\
0, & H_{R}
\end{array},\right. \\
& \tilde{f}_{\omega l}^{\mathrm{R}}=\left\{\begin{array}{cc}
0 & H_{L} \\
e^{-i \omega v}, & H_{R}
\end{array}\right.
\end{aligned}
$$


We will use these modes repeatedly later in the paper. Note, however, that we do not involve these modes in a definition of a quantum state.

Let us now turn to the definition of the Unruh state [28]. The field operator is decomposed as in Eq. 2.3 and the modes [satisfying Eq. (2.4)] as in Eq. 2.5. Everything remains the same except for the initial conditions for the modes $\tilde{g}_{\omega l}^{\Lambda}$ (where we use the letter $g$ for the Unruh modes) that now take the form

$$
\tilde{g}_{\omega l}^{\mathrm{up}}=\left\{\begin{array}{rrr}
e^{-i \omega U}, & H_{\text {past }} \cup H_{L} \\
0 & , & \text { PNI }
\end{array}\right.
$$

and

$$
\tilde{g}_{\omega l}^{\text {in }}=\left\{\begin{array}{cc}
0, & H_{\text {past }} \cup H_{L} \\
e^{-i \omega v}, & \text { PNI }
\end{array} .\right.
$$

Using the decomposition 2.3 but with the Unruh modes defined above, the Unruh state is defined as in Eq. 2.11. Explicitly, if we decompose the scalar field operator $\hat{\Phi}(x)$ as

$$
\hat{\Phi}(x)=\int d \omega \sum_{\Lambda, l, m}\left[g_{\omega l m}^{\Lambda}(x) \hat{b}_{\omega l m}^{\Lambda}+g_{\omega l m}^{\Lambda *}(x) \hat{b}_{\omega l m}^{\Lambda \dagger}\right]
$$

the Unruh state is defined by

$$
\hat{b}_{\omega l m}^{\Lambda}|0\rangle_{U}=0
$$

Notice that the Unruh modes (and quantum state) are defined both in the interior and the exterior regions of the $\mathrm{BH}$ [regions I and II of Fig. [1]].

In Unruh state, the expectation value of the stress-energy tensor corresponds to the Hawking radiation at infinity and it is regular, in a freely falling frame, on the future event horizon but not on the past horizon [27.

Finally, the third quantum state that we consider is the Hartle-Hawking state [29]. It is usually defined by an analytic continuation to the Euclidean sector, but we will mainly be interested in the mode structure of the state, as we used above for the Unruh and Boulware states. In fact, we shall be interested in known mode-sum expressions for various expectation values at the Hartle-Hawking state and will not need to use the precise form of the modes themselves, see below. The expectation value of the stress-energy tensor in Hartle-Hawking state corresponds to a thermal bath of radiation at infinity and it is regular, in a freely falling frame, on both of the event horizons [27].

This state is denoted by $|0\rangle_{H}$.

\section{B. Regularization and Renormalization}

In this paper, we consider two kinds of composite operators, $\hat{\Phi}^{2}(x)$ and $\hat{T}_{\mu \nu}(x)$, which are quadratic in the scalar field operator and its derivatives. As is well known, composite operators formally contain divergences and need to be renormalized in order to yield a well-defined quantity. In this paper, we follow the renormalization procedure initiated by Candelas in [27] and recently further analyzed (and generalized to a much larger extent) in [30 33. This procedure is based on the so called point-splitting method. We begin this subsection with briefly reviewing the point-splitting method and then continue with a description of the pragmatic renormalization procedure used in this paper.

\section{Point-Splitting}

When dealing with composite operators which are quadratic in the field operator and its derivatives, an intuitive way to regularize these operators is to "split" the point $x$ to two distinct points $x$ and $x^{\prime}$, and consider the operator which is the product of the two constituent operators at the two different points. This operator is obviously welldefined. Next, we can subtract a counterterm that removes the singularity and take the coincidence limit $x^{\prime} \rightarrow x$, thereby obtaining the renormalized operator. This is the so called point-splitting method. An essential property of the counterterm is that it is a local geometric quantity that fully captures the singular piece. In other words, it is independent of the quantum state (and therefore we can write the renormalization as an operator equation). Moreover, note that if we consider the vacuum expectation value of the split operator, we get the standard two-point function. 
In Refs. [34, 35, Christensen used the DeWitt-Schwinger expansion of the Feynman Green's function [36, 37] (see also [38]) to obtain the counterterms needed for the renormalization of $\left\langle\hat{\Phi}^{2}\right\rangle$ and $\left\langle\hat{T}_{\mu \nu}\right\rangle$ in the point-splitting method. In the case of $\hat{\Phi}^{2}$, the result is

$$
\left\langle\hat{\Phi}^{2}(x)\right\rangle_{r e n}=\lim _{x^{\prime} \rightarrow x}\left[\left\langle\hat{\Phi}(x) \hat{\Phi}\left(x^{\prime}\right)\right\rangle-G_{D S}\left(x, x^{\prime}\right)\right],
$$

where $G_{D S}\left(x, x^{\prime}\right)$ is known as the DeWitt-Schwinger counterterm, which for a scalar field with mass $m$ and coupling constant $\xi$ takes the form [16, 34]

$$
G_{D S}\left(x, x^{\prime}\right)=\frac{1}{8 \pi^{2} \sigma}+\frac{m^{2}+(\xi-1 / 6) R}{8 \pi^{2}}\left[\gamma+\frac{1}{2} \ln \left(\frac{\mu^{2}|\sigma|}{2}\right)\right]-\frac{m^{2}}{16 \pi^{2}}+\frac{1}{96 \pi^{2}} R_{\mu \nu} \frac{\sigma^{; \mu} \sigma^{; \nu}}{\sigma} .
$$

Here, $\sigma$ is the biscalar of geodetic separation (also known as Synge's world function) which is equal to one-half of the square of the geodesic distance between $x$ and $x^{\prime}, \gamma$ is the Euler constant and $R_{\mu \nu}$ and $R$ are the Ricci tensor and scalar, respectively. The parameter $\mu$ is not uniquely fixed and corresponds to the well-known ambiguity in the renormalization procedure.

For the stress-energy tensor, the procedure is analogous and we have

$$
\left\langle\hat{T}_{\mu \nu}(x)\right\rangle_{r e n}=\lim _{x^{\prime} \rightarrow x}\left[\left\langle\hat{T}_{\mu \nu}\left(x, x^{\prime}\right)\right\rangle-C_{\mu \nu}^{D S}\left(x, x^{\prime}\right)\right],
$$

where $C_{\mu \nu}^{D S}\left(x, x^{\prime}\right)$ is the corresponding counterterm, which again is a local geometric quantity built from $\sigma$ and the metric. In Christensen's original prescription [35], the expression $\left\langle\hat{T}_{\mu \nu}\left(x, x^{\prime}\right)\right\rangle$, to which we may call the split stressenergy tensor, involves covariant derivatives taken at both $x$ and $x^{\prime}$, along with the bi-vector of parallel transport which transfers a vector at $x$ to a vector at $x^{\prime}$. In this paper, however, we use the alternative form of this prescription found in [33]. In this new form, all the derivatives in $\left\langle\hat{T}_{\mu \nu}\left(x, x^{\prime}\right)\right\rangle$ are taken at the same point $x$, and the bi-vector of parallel transport only appears in the new form of the counterterm. This way, the derivation of the mode-sum expression for $\left\langle\hat{T}_{\mu \nu}\left(x, x^{\prime}\right)\right\rangle$ is much easier and one can naively apply the classical expressions for the stress-energy tensor for the relevant modes in order to get this mode-sum. For further discussion and the explicit form of this new counterterm $C_{\mu \nu}^{D S}\left(x, x^{\prime}\right)$, see [33].

\section{Pragmatic mode-sum renormalization method}

Implementing the point-splitting renormalization is most easily done using the method introduced in 27, 30 33. In this method, we first use the mode decomposition of the field operator in order to get a mode-sum expression for the split operator (e.g. the two-point function in the case of $\left\langle\hat{\Phi}^{2}\right\rangle$ ). The splitting is done along a direction of a symmetry of the geometry, i.e. in the direction of a Killing vector. Then, we write the counterterm as an integral which is of the same kind as one of those used in the mode-sum expression (this integral is done over a variable which is conjugate to the symmetry direction coordinate), and subtract the two. After doing that, we can take the coincidence limit and get the result (up to some extra regularizations that might be needed). The surprising thing about this procedure is that one can actually take the coincidence limit right at the beginning. In other words, one can write a formal mode-sum expression for the expectation value under consideration $\left(\right.$ e.g. $\left.\left\langle\hat{\Phi}^{2}\right\rangle\right)$, which is a divergent quantity, and after doing the same for the counterterm, subtract the two. The quantity obtained in this way might still be a divergent one, but after removing these nonphysical divergences one gets the correct result.

In this paper we will only be concerned with a simple application of this renormalization method. Many of the subtleties that arise in other cases will not occur in our case. The results are derived using the $t$-splitting variant of the method (see [30, 33]), but they were also confirmed using the $\theta$-splitting variant (see [31]).

\section{The trace of the stress-energy tensor}

When considering a theory with a conformal symmetry, such as a massless, conformally coupled scalar field, the trace of the stress-energy tensor operator is a local, geometric quantity (independent of the quantum state) which is given by [38]

$$
T_{\text {anomaly }} \equiv \frac{1}{2880 \pi^{2}}\left(R_{\alpha \beta \gamma \delta} R^{\alpha \beta \gamma \delta}-R_{\alpha \beta} R^{\alpha \beta}+\frac{5}{2} R^{2}+6 \square R\right)
$$


This result can be generalized to a nonconformal theory as was shown in [24] and rewritten in a more convenient form for our analysis in [25]. In this paper, we consider a massless, minimally coupled scalar field, for which we have the following result:

$$
\left\langle\hat{T}_{\mu}^{\mu}\right\rangle_{\text {ren }}+\frac{1}{2} \square\left\langle\hat{\Phi}^{2}\right\rangle_{\text {ren }}=T_{\text {anomaly }},
$$

relating the expectation values of the two quantities we calculate.

\section{EXPECTATION VALUE OF THE STRESS-ENERGY TENSOR NEAR THE EVENT HORIZON}

Before turning to the calculation of the stress-energy tensor near the inner horizon, we begin by applying our analytical approach to the calculation of the leading-order divergence of $\left\langle\hat{\Phi}^{2}\right\rangle_{r e n}$ and $\left\langle\hat{T}_{\mu \nu}\right\rangle_{r e n}$ in Boulware state upon approaching the outer (event) horizon from outside of the BH. We consider a massless, minimally coupled scalar field as our quantum field. In general, the expectation value of the stress-energy tensor, evaluated in a freely falling frame, is known to be regular on both the past and future event (outer) horizons in the case of the Hartle-Hawking state, it is regular on the future horizon but not on the past horizon in the case of the Unruh state, and it diverges on both of the horizons in the case of the Boulware state [see the discussion in subSec. (II A) and Ref. [27] for the case of Schwarzschild spacetime].

In the present section, in contrast to the analysis in the interior of the $\mathrm{BH}$ (discussed in the rest of the paper), we will not renormalize the expectation values using the explicit form of the corresponding counterterms. Instead, we follow a technique presented in [27, 39] ${ }^{1}$ and use the fact that the counterterms are geometric quantities independent of the quantum state, and the fact that the expectation values diverge at the event horizon most rapidly in Boulware state, in order to obtain the leading behavior of the renormalized expectation values in this state using the following subtractions:

$$
\left\langle\hat{\Phi}^{2}(x)\right\rangle_{B, r e n} \cong\left\langle\hat{\Phi}^{2}(x)\right\rangle_{B, r e n}-\left\langle\hat{\Phi}^{2}(x)\right\rangle_{U, r e n}=\left\langle\hat{\Phi}^{2}(x)\right\rangle_{B}-\left\langle\hat{\Phi}^{2}(x)\right\rangle_{U}, r \rightarrow r_{+}
$$

and similarly

$$
\left\langle\hat{T}_{\mu \nu}(x)\right\rangle_{B, r e n} \cong\left\langle\hat{T}_{\mu \nu}(x)\right\rangle_{B, r e n}-\left\langle\hat{T}_{\mu \nu}(x)\right\rangle_{U, r e n}=\left\langle\hat{T}_{\mu \nu}(x)\right\rangle_{B}-\left\langle\hat{T}_{\mu \nu}(x)\right\rangle_{U}, r \rightarrow r_{+},
$$

where the subscripts $B$ and $U$ denote the Boulware and Unruh states, respectively.

In Refs. [27, 39], formal expressions for $\left\langle\hat{\Phi}^{2}\right\rangle$ and $\left\langle\hat{T}_{\mu \nu}\right\rangle$ (in the three quantum states discussed here) as mode-sums involving the Boulware modes [see subSec. [IIA] ] were found. Let us quote them here for future reference ${ }^{2}$. If we denote the Boulware modes by $f_{\omega l m}^{\text {up }}$ and $f_{\omega l m}^{\text {in }}$, we have for $\left\langle\hat{\Phi}^{2}\right\rangle$ the following expressions:

$$
\begin{gathered}
\left\langle\hat{\Phi}^{2}(x)\right\rangle_{B}=\int_{0}^{\infty} d \omega \sum_{l, m}\left[\left|f_{\omega l m}^{\mathrm{up}}(x)\right|^{2}+\left|f_{\omega l m}^{\mathrm{in}}(x)\right|^{2}\right], \\
\left\langle\hat{\Phi}^{2}(x)\right\rangle_{U}=\int_{0}^{\infty} d \omega \sum_{l, m}\left[\operatorname{coth}\left(\frac{\pi \omega}{\kappa_{+}}\right)\left|f_{\omega l m}^{\mathrm{up}}(x)\right|^{2}+\left|f_{\omega l m}^{\mathrm{in}}(x)\right|^{2}\right], \\
\left\langle\hat{\Phi}^{2}(x)\right\rangle_{H}=\int_{0}^{\infty} d \omega \sum_{l, m} \operatorname{coth}\left(\frac{\pi \omega}{\kappa_{+}}\right)\left[\left|f_{\omega l m}^{\mathrm{up}}(x)\right|^{2}+\left|f_{\omega l m}^{\mathrm{in}}(x)\right|^{2}\right],
\end{gathered}
$$

\footnotetext{
${ }^{1}$ Note, however, that in these references the scalar field is conformally coupled instead of minimally coupled.

2 Notice that Refs. 27, 39] considered Schwarzschild spacetime and a conformally coupled scalar field, while we are interested in ReissnerNordström spacetime and a minimally coupled scalar field. However, there is no serious qualitative difference between the two for this analysis (carried out in the exterior region of the $\mathrm{BH}$ ), and the expressions map into each other under $\kappa \leftrightarrow \kappa_{+}$, where $\kappa$ is the surface gravity of the $\mathrm{BH}$, and under a suitable change in the functional form of the integrand in the mode-sum expression for the stress-energy tensor.
} 
where again the subscripts $B, U$ and $H$ denote the Boulware, Unruh and Hartle-Hawking states, respectively. For the stress-energy tensor $\left\langle\hat{T}_{\mu \nu}\right\rangle$, we similarly have

$$
\begin{gathered}
\left\langle\hat{T}_{\mu \nu}(x)\right\rangle_{B}=\int_{0}^{\infty} d \omega \sum_{l, m}\left\{T_{\mu \nu}\left[f_{\omega l m}^{\text {up }}(x), f_{\omega l m}^{\text {up } *}(x)\right]+T_{\mu \nu}\left[f_{\omega l m}^{\text {in }}(x), f_{\omega l m}^{\text {in } *}(x)\right]\right\}, \\
\left\langle\hat{T}_{\mu \nu}(x)\right\rangle_{U}=\int_{0}^{\infty} d \omega \sum_{l, m}\left\{\operatorname{coth}\left(\frac{\pi \omega}{\kappa_{+}}\right) T_{\mu \nu}\left[f_{\omega l m}^{\text {up }}(x), f_{\omega l m}^{\text {up } *}(x)\right]+T_{\mu \nu}\left[f_{\omega l m}^{\text {in }}(x), f_{\omega l m}^{\text {in } *}(x)\right]\right\}, \\
\left\langle\hat{T}_{\mu \nu}(x)\right\rangle_{H}=\int_{0}^{\infty} d \omega \sum_{l, m} \operatorname{coth}\left(\frac{\pi \omega}{\kappa_{+}}\right)\left\{T_{\mu \nu}\left[f_{\omega l m}^{\text {up }}(x), f_{\omega l m}^{\text {up } *}(x)\right]+T_{\mu \nu}\left[f_{\omega l m}^{\text {in }}(x), f_{\omega l m}^{\text {in } *}(x)\right]\right\},
\end{gathered}
$$

where

$$
T_{\mu \nu}\left[f, f^{*}\right]=\frac{1}{2}\left(f_{, \mu} f_{, \nu}^{*}+f_{, \nu} f_{, \mu}^{*}-g_{\mu \nu} g^{\alpha \beta} f_{, \alpha} f_{, \beta}^{*}\right)
$$

Substituting the above expressions for $\left\langle\hat{\Phi}^{2}\right\rangle$ and $\left\langle\hat{T}_{\mu \nu}\right\rangle$ in the different states into Eqs. 3.1) and 3.2), we get the following mode-sums for the leading asymptotic behavior of $\left\langle\hat{\Phi}^{2}\right\rangle_{B, \text { ren }}$ and $\left\langle\hat{T}_{\mu \nu}\right\rangle_{B \text {,ren }}$ on approaching the outer (event) horizon from outside of the BH:

$$
\left\langle\hat{\Phi}^{2}(x)\right\rangle_{B, r e n} \cong-2 \int_{0}^{\infty} \frac{d \omega}{e^{2 \pi \omega / \kappa_{+}-1}} \sum_{l, m}\left|f_{\omega l m}^{\mathrm{up}}(x)\right|^{2}, r \rightarrow r_{+}
$$

and

$$
\left\langle\hat{T}_{\mu \nu}(x)\right\rangle_{B, r e n} \cong-2 \int_{0}^{\infty} \frac{d \omega}{e^{2 \pi \omega / \kappa_{+}-1}} \sum_{l, m} T_{\mu \nu}\left[f_{\omega l m}^{\text {up }}(x), f_{\omega l m}^{\text {up } *}(x)\right], r \rightarrow r_{+} \cdot
$$

Next, using an asymptotic calculation of the "up" Boulware modes $f_{\omega l m}^{\text {up }}$ near the event horizon, we will find the explicit asymptotic behavior of $\left\langle\hat{\Phi}^{2}\right\rangle_{B \text {, ren }}$ and $\left\langle\hat{T}_{\mu \nu}\right\rangle_{B \text {, ren }}$ by substitution of these modes into Eqs. 3.9. and 3.10. In order to do it, we begin by exploiting the time-translational symmetry and write $\tilde{f}_{\omega l}^{\Lambda}$ [recall that $\tilde{f}_{\omega l}^{\Lambda}$ is related to $f_{\omega l m}^{\Lambda}$ via Eq. (2.5)] as a product of a simple time-dependent part (common to the "up" and "in" modes) and a radial function as follows:

$$
\tilde{f}_{\omega l}^{\Lambda}(r, t)=e^{-i \omega t} \Psi_{\omega l}^{\Lambda}(r)
$$

By substituting into Eq. 2.7), we get the following equation for the radial functions:

$$
\Psi_{\omega l, r_{*} r_{*}}^{\Lambda}+\left(\omega^{2}-V_{l}(r)\right) \Psi_{\omega l}^{\Lambda}=0 .
$$

The asymptotic forms of these functions are easily determined from those of $\tilde{f}_{\omega l}^{\Lambda}$ [given in Eqs. 2.9] and [2.10] and are given by

$$
\Psi_{\omega l}^{\mathrm{in}}(r) \cong\left\{\begin{array}{c}
\tau_{\omega l}^{\mathrm{in}} e^{-i \omega r_{*}}, \quad r_{*} \rightarrow-\infty \\
e^{-i \omega r_{*}}+\rho_{\omega l}^{\mathrm{in}} e^{i \omega r_{*}}, \quad r_{*} \rightarrow \infty
\end{array}\right.
$$

and

$$
\Psi_{\omega l}^{\mathrm{up}}(r) \cong\left\{\begin{array}{cc}
e^{i \omega r_{*}}+\rho_{\omega l}^{\mathrm{up}} e^{-i \omega r_{*}} \quad, \quad r_{*} \rightarrow-\infty \\
\tau_{\omega l}^{\mathrm{up}} e^{i \omega r_{*}}, \quad r_{*} \rightarrow \infty
\end{array}\right.
$$

where $\rho_{\omega l}^{\Lambda}$ and $\tau_{\omega l}^{\Lambda}$ are the reflection and transmission coefficients (corresponding to the mode $\Lambda$ ), respectively. 
Now, since we want to find the asymptotic form of $f_{\omega l m}^{\text {up }}$ near the event horizon, we concentrate on finding the asymptotic form of $\Psi_{\omega l}^{\mathrm{up}}(r)$ near $r_{*} \rightarrow-\infty$. For that, we expand the potential (2.8) near $r=r_{+}\left(\right.$or $\left.r_{*} \rightarrow-\infty\right)$ and get the asymptotic form

$$
V_{l}(r) \cong C_{+}\left(r-r_{+}\right) \cong C_{+}\left(r_{+}-r_{-}\right) \exp \left(-2 \kappa_{+} r_{+}\right) \exp \left(2 \kappa_{+} r_{*}\right), r_{*} \rightarrow-\infty,
$$

where

$$
C_{+}=\frac{r_{+}-r_{-}}{r_{+}^{4}}\left(l^{2}+l+1-\frac{r_{-}}{r_{+}}\right) .
$$

Substituting this potential into Eq. 3.12 , we find the following asymptotic form of the radial equation near $r=r_{+}$:

$$
R_{\omega l, r_{*} r_{*}}+\left[\omega^{2}-C_{+}\left(r_{+}-r_{-}\right) \exp \left(-2 \kappa_{+} r_{+}\right) \exp \left(2 \kappa_{+} r_{*}\right)\right] R_{\omega l}=0,
$$

where $R_{\omega l}(r)$ denotes the asymptotic form of $\Psi_{\omega l}^{\mathrm{up}}(r)$ near $r=r_{+}$. Notice that the boundary condition (or asymptotic form) of $R_{\omega l}(r)$ at $r_{*} \rightarrow \infty$ is different from that of $\Psi_{\omega l}^{\mathrm{up}}(r)$, because we are considering an exponential potential in Eq. 3.14. We are looking for a solution $R_{\omega l}(r)$ that decays exponentially at $r_{*} \rightarrow \infty$ and takes the form $e^{i \omega r_{*}}+\rho_{\omega l}^{\mathrm{up}} e^{-i \omega r_{*}}$ at $r_{*} \rightarrow-\infty$. The solution that satisfies these conditions is

$$
R_{\omega l}\left(r_{*}\right)=\left(\frac{C_{+} r_{+}^{4}}{r_{+}-r_{-}}\right)^{-i \omega /\left(2 \kappa_{+}\right)} \frac{2 \exp \left(i \omega r_{+}\right)}{\Gamma\left(-i \omega / \kappa_{+}\right)} K_{i \omega / \kappa_{+}}\left(2 r_{+}^{2} \sqrt{\frac{C_{+}}{r_{+}-r_{-}}} e^{\kappa_{+}\left(r_{*}-r_{+}\right)}\right),
$$

where $K_{\alpha}(z)$ is a modified Bessel function of the second kind. We can easily write this expression in terms of $r$ by noticing that at $r_{*} \rightarrow-\infty$ we have (recall that we are only interested in $R_{\omega l}$ in this asymptotic region)

$$
e^{\kappa_{+}\left(r_{*}-r_{+}\right)} \cong\left(\frac{r-r_{+}}{r_{+}-r_{-}}\right)^{1 / 2}, r \rightarrow r_{+} .
$$

In the rest of the calculation, we make the assumption that the leading divergence we are calculating results from large- $l$ values. In addition to the match between the analytical expressions thus derived and numerical results (see below), one can motivate this assumption using the following heuristic argument. First, we notice that the mode-sums (3.9) and (3.10) contain the factor $\left(e^{2 \pi \omega / \kappa_{+}}-1\right)^{-1}$ which decays exponentially at large $\omega$. As a result, the dominant contribution to the integral over $\omega$ comes from small- $\omega$ values (up to the scale $\kappa_{+} / 2 \pi \omega$ ). Next, since at large- $l$ values (and fixed $r$ ) the redial functions $\Psi_{\omega l}^{\Lambda}$ decay exponentially with $l$ according to the WKB approximation to the solution of Eq. (3.12) (recall that $\omega$ is small), the dominant contribution to the sum over $l$ comes from $l$-values that satisfy [see the discussion about the WKB method in subSec. [VB]

$$
l \leq \frac{r^{2}}{M \sqrt{\left(r-r_{+}\right)\left(r-r_{-}\right)}} \equiv l_{\mathrm{Max}}
$$

At each $r>r_{+}, l_{\text {Max }}$ is finite and so is the mode-sum; however, at the event horizon $\left(r=r_{+}\right) l_{\text {Max }}$ diverges and we get an infinite contribution to the mode-sum. This suggests that the leading (in $r-r_{+}$) divergence we wish to calculate is a large- $l$ effect and we assume that it is obtained from the leading large- $l$ behavior. Therefore, we take this limit of $R_{\omega l}$. It is more convenient to work with the variable

$$
\tilde{l}=l+\frac{1}{2}
$$

instead of $l$, and so we write $R_{\omega l}$ in terms of $\tilde{l}$ and take the asymptotic large- $\tilde{l}$ form of it. Using

$$
r_{+}^{2} \sqrt{\frac{C_{+}}{r_{+}-r_{-}}}=\tilde{l}+\mathcal{O}\left(\tilde{l}^{-1}\right)
$$

we get (writing the expression in terms of $r$ )

$$
R_{\omega l}(r) \cong \tilde{l}^{-i \omega / \kappa_{+}} \frac{2 \exp \left(i \omega r_{+}\right)}{\Gamma\left(-i \omega / \kappa_{+}\right)} K_{i \omega / \kappa_{+}}\left[2 \tilde{l}\left(\frac{r-r_{+}}{r_{+}-r_{-}}\right)^{1 / 2}\right]
$$


Recalling that as $r_{*} \rightarrow-\infty$ this radial function should take the form $e^{i \omega r_{*}}+\rho_{\omega l}^{\mathrm{up}} e^{-i \omega r_{*}}$, we get the following expression for $\rho_{\omega l}^{\text {up }}$ (to leading order in $\left.\tilde{l}\right)$ :

$$
\rho_{\omega l}^{\mathrm{up}} \cong \tilde{l}^{-2 i \omega / \kappa_{+}} e^{2 i \omega r_{+}} \frac{\Gamma\left(i \omega / \kappa_{+}\right)}{\Gamma\left(-i \omega / \kappa_{+}\right)},
$$

where $\Gamma(x)$ is the Euler gamma function.

Now that we found the asymptotic form of $\Psi_{\omega l}^{\mathrm{up}}(r)$ near $r_{+}\left(\right.$recall that $\left.\Psi_{\omega l}^{\mathrm{up}}(r) \cong R_{\omega l}(r)\right)$, we have the asymptotic form of $f_{\omega l m}^{\text {up }}$ using Eqs. (3.11) and 2.5. Substituting it to Eqs. (3.9) and (3.10) yields the leading asymptotic behavior of $\left\langle\hat{\Phi}^{2}\right\rangle_{B, \text { ren }}$ and $\left\langle\hat{T}_{\mu \nu}\right\rangle_{B, \text { ren }}$ on approaching the outer (event) horizon of the $\mathrm{BH}^{3}$ :

$$
\left\langle\hat{\Phi}^{2}(x)\right\rangle_{B, r e n} \cong-\frac{\kappa_{+}}{96 \pi^{2}\left(r-r_{+}\right)}, r \rightarrow r_{+}
$$

and

$$
\left\langle\hat{T}_{\nu}^{\mu}(x)\right\rangle_{B, \text { ren }} \cong \frac{\kappa_{+}^{2}}{5760 \pi^{2}\left(r-r_{+}\right)^{2}}\left(\begin{array}{cccc}
33 & 0 & 0 & 0 \\
0 & -11 & 0 & 0 \\
0 & 0 & 19 & 0 \\
0 & 0 & 0 & 19
\end{array}\right), r \rightarrow r_{+} .
$$

Note that the stress-energy tensor is presented in a mixed form and in Schwarzschild coordinates. A straightforward calculation reveals that this expression for the stress-energy tensor satisfies the energy-momentum conservation equation at leading order. Moreover, these two expressions for $\left\langle\hat{\Phi}^{2}\right\rangle_{B, \text { ren }}$ and $\left\langle\hat{T}_{\mu \nu}\right\rangle_{B \text {,ren }}$ satisfy Eq. 2.20 at leading order and correspond to previous numerical results obtained in [16, 17.

We obtained that the expectation values of $\hat{\Phi}^{2}$ and $\hat{T}_{\mu \nu}$ in Boulware state diverge at the event horizon. We shall proceed to analyze the expectation values of these operators in Unruh and Hartle-Hawking states in the interior region of the $\mathrm{BH}$.

\section{THE TWO-POINT FUNCTION IN THE INTERIOR REGION OF THE BLACK HOLE}

In the previous section, we looked at the exterior region of the BH and used mode-sum expressions for $\left\langle\hat{\Phi}^{2}\right\rangle$ and $\left\langle\hat{T}_{\mu \nu}\right\rangle$ [see Eqs. [3.3)-(3.8)] in terms of the Boulware modes (or outer Eddington-Finkelstein modes) in order to find their asymptotic behavior on approaching the event (outer) horizon. The season for using the Boulware modes was the simple equation satisfied by the radial functions of these modes. Finding the asymptotic form of these radial functions enabled us to get the desired asymptotic behavior of $\left\langle\hat{\Phi}^{2}\right\rangle$ and $\left\langle\hat{T}_{\mu \nu}\right\rangle$.

In order to investigate the leading-order behavior of $\left\langle\hat{\Phi}^{2}\right\rangle$ and $\left\langle\hat{T}_{\mu \nu}\right\rangle$ near the inner horizon, we will use the same technique. That is, we will use mode-sum expressions in terms of the inner (instead of outer) Eddington-Finkelstein modes, and after finding the asymptotic form of their radial functions, we will get the asymptotic behavior of $\left\langle\hat{\Phi}^{2}\right\rangle$ and $\left\langle\hat{T}_{\mu \nu}\right\rangle$. It is important to note, however, that even though the general idea of the calculation is the same as that of the one carried out in the exterior region, many things are different. For example, the potential that appears in the radial equation is no longer positive, a fact that influences the analysis of the radial functions inside the $\mathrm{BH}$. Moreover, we will not use subtractions as in Eqs. 3.1) and (3.2). Instead, we will use explicit renormalization using counterterms.

In this section, we review the form of the mode-sum expression for the two-point function in the interior region found in Ref. [25]. The mode-sum is in terms of the inner Eddington-Finkelstein modes, defined in Eqs. (2.12), (2.13) and 2.5p. After taking the coincidence limit, one gets $\left\langle\hat{\Phi}^{2}\right\rangle .\left\langle\hat{T}_{\mu \nu}\right\rangle$ can also be obtained after the application of a certain differential operator ${ }^{4}$. The focus in [25] is on the symmetrized form of the two-point function, which is also known as the Hadamard elementary function and is defined by

$$
G^{(1)}\left(x, x^{\prime}\right)=\left\langle\left\{\hat{\Phi}(x), \hat{\Phi}\left(x^{\prime}\right)\right\}\right\rangle .
$$

\footnotetext{
${ }^{3}$ In these calculations we took advantage of the fact that the leading contribution comes from large- $l$ values and switched the summation over $l$ with an integral.

${ }^{4}$ Note that if one only takes the coincidence limit, the result is a formal mode-sum expression for $<\hat{\Phi}^{2}>$ and $<\hat{T}_{\mu \nu}>$. If, on the other hand, one subtracts the corresponding counterterms in the process, the result is the renormalized forms.
} 
There, it was found that in the Unruh state we have

$$
\begin{gathered}
G_{U}^{(1)}\left(x, x^{\prime}\right)=\sum_{l, m} \int_{0}^{\infty} d \omega\left[\operatorname{coth}\left(\frac{\pi \omega}{\kappa_{+}}\right)\left\{f_{\omega l m}^{\mathrm{L}}(x), f_{\omega l m}^{\mathrm{L} *}\left(x^{\prime}\right)\right\}+\left(\operatorname{coth}\left(\frac{\pi \omega}{\kappa_{+}}\right)\left|\rho_{\omega l}^{\mathrm{up}}\right|^{2}+\left|\tau_{\omega l}^{\mathrm{up}}\right|^{2}\right)\left\{f_{\omega l m}^{\mathrm{R}}(x), f_{\omega l m}^{\mathrm{R} *}\left(x^{\prime}\right)\right\}\right. \\
\left.+2 \operatorname{csch}\left(\frac{\pi \omega}{\kappa_{+}}\right) \operatorname{Re}\left(\rho_{\omega l}^{\mathrm{up}}\left\{f_{\omega l m}^{\mathrm{R}}(x), f_{-\omega l m}^{\mathrm{L} *}\left(x^{\prime}\right)\right\}\right)\right]
\end{gathered}
$$

where $\rho_{\omega l}^{\text {up }}$ and $\tau_{\omega l}^{\text {up }}$ are the reflection and transmission coefficients that correspond to the "up" Boulware mode (in the exterior region), respectively. We define curly brackets acting on functions as a symmetrization with respect to the arguments of these functions, i.e.

$$
\left\{A(x), B\left(x^{\prime}\right)\right\}=A(x) B\left(x^{\prime}\right)+A\left(x^{\prime}\right) B(x) .
$$

In the Hartle-Hawking state, the Hadamard function takes the form

$$
\begin{gathered}
G_{H}^{(1)}\left(x, x^{\prime}\right)=\sum_{l, m} \int_{0}^{\infty} d \omega\left[\operatorname{coth}\left(\frac{\pi \omega}{\kappa_{+}}\right)\left(\left\{f_{\omega l m}^{\mathrm{L}}(x), f_{\omega l m}^{\mathrm{L} *}\left(x^{\prime}\right)\right\}+\left\{f_{\omega l m}^{\mathrm{R}}(x), f_{\omega l m}^{\mathrm{R} *}\left(x^{\prime}\right)\right\}\right)\right. \\
\left.+2 \operatorname{csch}\left(\frac{\pi \omega}{\kappa_{+}}\right) \operatorname{Re}\left(\rho_{\omega l}^{\mathrm{up}}\left\{f_{\omega l m}^{\mathrm{R}}(x), f_{-\omega l m}^{\mathrm{L} *}\left(x^{\prime}\right)\right\}\right)\right] .
\end{gathered}
$$

As mentioned above, from these Hadamard functions one can obtain $\left\langle\hat{\Phi}^{2}\right\rangle$ and $\left\langle\hat{T}_{\mu \nu}\right\rangle$ in the respective states.

Next, as we did in the last section [Sec. [III]] in the case of the exterior region, we decompose $\tilde{f}_{\omega l}^{\mathrm{L}}$ and $\tilde{f}_{\omega l}^{\mathrm{R}}[\mathrm{related}$ to $f_{\omega l m}^{\mathrm{L}}$ and $f_{\omega l m}^{\mathrm{R}}$ through Eq. [2.5] ] into a time-dependent part and a radial function. In contrast to the exterior region, in the interior the spacelike and timelike nature of the coordinates $t$ and $r_{*}$ is interchanged. As a result, in the case of the interior we use the following decomposition

$$
\tilde{f}_{\omega l}^{\mathrm{L}}(r, t)=e^{i \omega t} \psi_{\omega l}(r), \quad \tilde{f}_{\omega l}^{\mathrm{R}}(r, t)=e^{-i \omega t} \psi_{\omega l}(r)
$$

in terms of a single radial function and two different time-dependent parts. The radial function $\psi_{\omega l}$ satisfies Eq. 3.12 and its boundary condition is easily determined from Eqs. 2.12 and 2.13 to be

$$
\psi_{\omega l}(r) \cong e^{-i \omega r_{*}} \quad, \quad r_{*} \rightarrow-\infty
$$

Recall that $r_{*} \rightarrow-\infty$ corresponds to $r \rightarrow r_{+}$and that $t$ and $r_{*}$ are related to $v$ and $u_{\text {int }}$ (in the interior region) through Eq. 2.2.

Our main remaining task is to find the asymptotic form of the radial function $\psi_{\omega l}$ near the inner horizon. Then, using Eqs. 4.1, 4.2, 2.5 and 4.3 we will get the asymptotic behavior of $\left\langle\hat{\Phi}^{2}\right\rangle$ and $\left\langle\hat{T}_{\mu \nu}\right\rangle$ near that horizon.

\section{THE RADIAL FUNCTION IN THE INTERIOR REGION OF THE BLACK HOLE}

We now turn to the discussion of the radial function $\psi_{\omega l}$ in the interior region. Let us remind that this function satisfies Eq. (3.12 along with the boundary condition given in Eq. (4.4). Our goal is to find its asymptotic form near the inner horizon. For that, we divide the interior region $r_{-}<r<r_{+}$into three domains and use different methods in order to get approximations for the radial function in these domains. By matching the three expressions together we will be able to get the desired asymptotic form near the inner horizon [corresponding to the boundary condition in Eq. 4.4]. As in Sec. (III), we work in terms of $\tilde{l}$ [defined in Eq. (3.15)] and take the leading-order behavior in it. We assume that as in the case of the Boulware state in the external region, the asymptotic forms of the quantities we consider near the inner horizon are determined by this large- $\tilde{l}$ limit (even though the integrand structures of the two mode-sums are different). This assumption is consistent with numerical results that will be published elsewhere [18. Explicitly, we assume that for each radial function $\tilde{l}$ satisfy

$$
\tilde{l} \gg \omega M, \frac{r_{+}}{M} .
$$




\section{A. Region I $\left(r \rightarrow r_{+}\right)$}

Here we consider the vicinity of the outer horizon. We already looked at a similar limit in Sec. (III), but there we considered the exterior region and here we look at the interior. The expansion of the potential given in Eq. 3.13 remains the same, but since we are inside the BH, $r<r_{+}$, the potential is now negative (instead of positive) and the asymptotic form of the radial equation [given by Eq. (3.14) for the case of the exterior region] becomes

$$
\psi_{\omega l}^{I}, r_{*} r_{*}+\left[\omega^{2}+C_{+}\left(r_{+}-r_{-}\right) \exp \left(-2 \kappa_{+} r_{+}\right) \exp \left(2 \kappa_{+} r_{*}\right)\right] \psi_{\omega l}^{I}=0
$$

We are looking for a solution that satisfies the boundary condition given in Eq. 4.4. This solution, taken to leading order in $\tilde{l}$, is given by

$$
\psi_{\omega l}^{I}(r) \cong \tilde{l}^{i \omega / \kappa_{+}} e^{-i \omega r_{+}} \Gamma\left(1-i \omega / \kappa_{+}\right) J_{-i \omega / \kappa_{+}}\left[2 \tilde{l}\left(\frac{r_{+}-r}{r_{+}-r_{-}}\right)^{1 / 2}\right],
$$

where $J_{\alpha}(z)$ is a Bessel function of the first kind. Another representation, which will turn out to be interesting below, takes the form

$$
\psi_{\omega l}^{I}(r)=p_{1}^{I} F^{I}(r)+p_{2}^{I} F^{I *}(r)
$$

where

$$
\begin{gathered}
F^{I}(r)=\frac{e^{-i \pi / 4}}{\pi} K_{i \omega / \kappa_{+}}\left[-2 i \tilde{l}\left(\frac{r_{+}-r}{r_{+}-r_{-}}\right)^{1 / 2}\right] \\
p_{1}^{I}=e^{-i \frac{\pi}{4}} \tilde{l}{ }^{i \omega / \kappa+} e^{-i \omega r_{+}} \Gamma\left(1-i \omega / \kappa_{+}\right) e^{-\frac{\pi \omega}{2 \kappa_{+}}}
\end{gathered}
$$

and

$$
p_{2}^{I}=e^{i \frac{\pi}{4}} \tilde{l}^{i \omega / \kappa_{+}} e^{-i \omega r_{+}} \Gamma\left(1-i \omega / \kappa_{+}\right) e^{\frac{\pi \omega}{2 \kappa+}}
$$

\section{B. Region II (The middle region)}

We would now like to find the form of the radial function in the region which is not asymptotically close to the outer (or inner) horizon. This region is defined as the one in which we can employ the WKB approximation to a good accuracy in the following way. Defining

$$
k(r) \equiv\left(\omega^{2}-V_{l}(r)\right)^{1 / 2}
$$

and using the fact that the scale of variation of the potential $V_{l}(r)$ is given by the mass of the BH $M$, we see from the radial equation (3.12) that the region (i.e. $r$ values) in which the (leading order) WKB method yields a good approximation for the radial function is given by

$$
k(r) M \gg 1 .
$$

Exploiting the fact that we only consider large- $\tilde{l}$ modes [see Eq. [5.1)], we can write the potential $V_{l}(r)$ [given in Eq. (2.8] to a leading order in $\tilde{l}$ in the following way:

$$
V_{l}(r) \cong\left(1-\frac{2 M}{r}+\frac{Q^{2}}{r^{2}}\right) \frac{\tilde{l}^{2}}{r^{2}}=-\frac{\tilde{l}^{2}}{r^{4}}\left(r_{+}-r\right)\left(r-r_{-}\right) .
$$

Note that the asymptotic form of the potential used in Eqs. (3.14) and 5.2 can be obtained from this form in the limit $r \rightarrow r_{+}$(and the large- $\tilde{l}$ limit of $C_{+}$). Another consequence of Eq. (5.1) is that we can neglect $\omega$ in the definition of $k(r)$, i.e.

$$
k(r) \cong\left|V_{l}(r)\right|^{1 / 2} \cong \frac{\tilde{l}}{r^{2}}\left[\left(r_{+}-r\right)\left(r-r_{-}\right)\right]^{1 / 2}
$$


Overall, we can re-write Eq. (5.8) as follows:

$$
\frac{\tilde{l} M}{r^{2}}\left[\left(r_{+}-r\right)\left(r-r_{-}\right)\right]^{1 / 2} \gg 1
$$

and define region II as the one in which Eq. (5.11) is satisfied.

Since we are only interested in the leading order in $l$, in this region we use the leading-order WKB form for the radial function. We therefore define

$$
\psi_{\omega l}^{I I}=k^{-1 / 2}(r)\left[a_{+} \exp \left(i \int k(r) d r_{*}\right)+a_{-} \exp \left(-i \int k(r) d r_{*}\right)\right],
$$

where the coefficients $a_{+}$and $a_{-}$are determined by matching $\Psi_{\omega l}^{I I}$ and $\Psi_{\omega l}^{I}$ (see below). The integral over $k(r)$ is readily calculated using Eq. 5.10 and we get

$$
\int k(r) d r_{*}=-\tilde{l} \arctan \left(\frac{r-M}{\left[\left(r_{+}-r\right)\left(r-r_{-}\right)\right]^{1 / 2}}\right) .
$$

As a result, we have

$$
\begin{gathered}
\psi_{\omega l}^{I I}=\frac{r}{\sqrt{\tilde{l}}}\left[\left(r_{+}-r\right)\left(r-r_{-}\right)\right]^{-1 / 4}\left\{a_{+} \exp \left[-i \tilde{l} \arctan \left(\frac{r-M}{\left[\left(r_{+}-r\right)\left(r-r_{-}\right)\right]^{1 / 2}}\right)\right]\right. \\
\left.+a_{-} \exp \left[i \tilde{l} \arctan \left(\frac{r-M}{\left[\left(r_{+}-r\right)\left(r-r_{-}\right)\right]^{1 / 2}}\right)\right]\right\} .
\end{gathered}
$$

We can now look at the limit $r_{*} \rightarrow-\infty$ of $\psi_{\omega l}^{I I}$ and compare it with the limit $r_{*} \rightarrow \infty$ of $\psi_{\omega l}^{I}$. The two expressions thus obtained should yield the same result for $\psi_{\omega l}$ (in this overlapping region) and serve as a way to obtain $a_{+}$and $a_{-}$. Matching the two expressions, we get (see the appendix for more details)

$$
a_{+}=\left(\frac{\kappa_{+}}{2 \pi}\right)^{1 / 2} i^{\tilde{l}-1} e^{i \frac{\pi}{4}} \tilde{l}^{i \omega / \kappa_{+}} e^{-i \omega r_{+}} \Gamma\left(1-i \omega / \kappa_{+}\right) e^{-\frac{\pi \omega}{2 \kappa_{+}}}
$$

and

$$
a_{-}=\left(\frac{\kappa_{+}}{2 \pi}\right)^{1 / 2}(-i)^{\tilde{l}} e^{i \frac{\pi}{4}} \tilde{l}^{i \omega / \kappa_{+}} e^{-i \omega r_{+}} \Gamma\left(1-i \omega / \kappa_{+}\right) e^{\frac{\pi \omega}{2 \kappa_{+}}}
$$

An interesting observation is that each exponential term in $\psi_{\omega l}^{I I}$ corresponds in the limit $r_{*} \rightarrow-\infty$ (discussed above) to one of the Bessel functions $K_{\alpha}(z)$ in the representation of $\psi_{\omega l}^{I}$ given in Eq. (5.4) (when taken in the limit $r_{*} \rightarrow \infty$ ). In other words, in the overlapping region each Bessel function term turns into an exponent term.

\section{Region III $\left(r \rightarrow r_{-}\right)$}

As in region I, we can find from the asymptotic form of the potential (now near $r_{-}$) the corresponding asymptotic form of the radial equation. Then, by finding a solution that matches to $\psi_{\omega l}^{I I}$ in the overlap between regions II and III, we would obtain $\psi_{\omega l}^{I I I}$, which is the asymptotic form of the radial function $\psi_{\omega l}$ near $r_{-}$and the desired result of this section.

Expanding the potential near $r_{-}$[as was done in subSec. (V A ) and in Sec. (III) near $\left.r_{+}\right]$, we get the following asymptotic form of the radial equation:

$$
\psi_{\omega l}^{I I I}, r_{*} r_{*}+\left[\omega^{2}-C_{-}\left(r_{+}-r_{-}\right) \exp \left(2 \kappa_{-} r_{-}\right) \exp \left(-2 \kappa_{-} r_{*}\right)\right] \psi_{\omega l}^{I I I}=0
$$

where

$$
C_{-}=-\frac{r_{+}-r_{-}}{r_{-}^{4}}\left(l^{2}+l+1-\frac{r_{+}}{r_{-}}\right)
$$


has the following leading behavior at large $l$ (or $\tilde{l})$ :

$$
C_{-} \cong-\frac{2 \kappa_{-}}{r_{-}^{2}} \tilde{l}^{2}
$$

This equation is analogous to Eq. (3.16) (but note that $C_{-}$is negative while $C_{+}$is positive).

The general solution (to leading order in $\tilde{l}$ ) to Eq. (5.16) can be written as

$$
\psi_{\omega l}^{I I I}=p_{1}^{I I I} F^{I I I}(r)+p_{2}^{I I I} F^{I I *}(r),
$$

where

$$
F^{I I I}(r)=\frac{e^{-i \pi / 4}}{\pi} K_{i \omega / \kappa_{-}}\left[-2 i \tilde{l}\left(\frac{r-r_{-}}{r_{+}-r_{-}}\right)^{1 / 2}\right] .
$$

As stated above, the coefficients $p_{1}^{I I I}$ and $p_{2}^{I I I}$ are determined by matching $\psi_{\omega l}^{I I I}$ in the limit $r_{*} \rightarrow-\infty$ with $\psi_{\omega l}^{I I}$ in the limit $r_{*} \rightarrow \infty$. The result is (see the appendix for more details)

$$
p_{1}^{I I I}=\frac{r_{-}}{r_{+}}(-1)^{\tilde{l}} e^{-i \frac{3 \pi}{4}} \tilde{l}^{i \omega / \kappa_{+}} e^{-i \omega r_{+}} \Gamma\left(1-i \omega / \kappa_{+}\right) e^{\frac{\pi \omega}{2 \kappa_{+}}}
$$

and

$$
p_{2}^{I I I}=\frac{r_{-}}{r_{+}}(-1)^{\tilde{l}} e^{-i \frac{\pi}{4}} \tilde{l}^{i \omega / \kappa_{+}} e^{-i \omega r_{+}} \Gamma\left(1-i \omega / \kappa_{+}\right) e^{-\frac{\pi \omega}{2 \kappa_{+}}} .
$$

Analogously to the observation made at the end of the last subsection, here also an exponential term in $\psi_{\omega l}^{I I}$ turns in the overlapping region (between regions II and III) into a Bessel function term in $\psi_{\omega l}^{I I I}$.

An alternative way to write $\psi_{\omega l}^{I I I}$ in terms of a different kind of Bessel function is as follows:

$$
\psi_{\omega l}^{I I I}=T h(r)+R h^{*}(r),
$$

where

$$
\begin{gathered}
h(r)=\tilde{l}^{-i \omega / \kappa_{-}} e^{-i \omega r_{-}} \Gamma\left(1+i \omega / \kappa_{-}\right) J_{i \omega / \kappa_{-}}\left[2 \tilde{l}\left(\frac{r-r_{-}}{r_{+}-r_{-}}\right)^{1 / 2}\right], \\
T=(-1)^{\tilde{l}-1 / 2}\left(\frac{r_{-}}{r_{+}}\right) \tilde{l}^{i \omega\left(\kappa_{+}^{-1}+\kappa_{-}^{-1}\right)} e^{-i \omega\left(r_{+}-r_{-}\right)} \frac{\Gamma\left(1-i \omega / \kappa_{+}\right)}{\Gamma\left(1+i \omega / \kappa_{-}\right)} \frac{\sinh \left[\frac{1}{2} \pi \omega\left(\kappa_{+}^{-1}+\kappa_{-}^{-1}\right)\right]}{\sinh \left(\pi \omega / \kappa_{-}\right)},
\end{gathered}
$$

and

$$
R=(-1)^{\tilde{l}+1 / 2}\left(\frac{r_{-}}{r_{+}}\right) \tilde{l}^{i \omega\left(\kappa_{+}^{-1}-\kappa_{-}^{-1}\right)} e^{-i \omega\left(r_{+}+r_{-}\right)} \frac{\Gamma\left(1-i \omega / \kappa_{+}\right)}{\Gamma\left(1-i \omega / \kappa_{-}\right)} \frac{\sinh \left[\frac{1}{2} \pi \omega\left(\kappa_{+}^{-1}-\kappa_{-}^{-1}\right)\right]}{\sinh \left(\pi \omega / \kappa_{-}\right)} .
$$

The advantage of this form is that the function $h(r)$ satisfies

$$
h\left(r_{*}\right) \cong e^{-i \omega r_{*}} \quad, \quad r_{*} \rightarrow \infty
$$

when viewed as a function of $r_{*}$. As a result, we have

$$
\psi_{\omega l}^{I I I} \cong T e^{-i \omega r_{*}}+R e^{i \omega r_{*}} \quad, \quad r_{*} \rightarrow \infty .
$$

Therefore, if we recall that the "initial condition" is given by Eq. (4.4), we see that we can regard $R$ and $T$ as reflection and transmission coefficients inside the $\mathrm{BH}$, respectively. 


\section{The Wronskian}

A nontrivial check for the above expressions for $\psi_{\omega l}$ in the different regions is the computation of the corresponding Wronskians. The Wronskian of Eq. 33.12 is conserved, and therefore we expect the Wronskians calculated with respect to $\psi_{\omega l}^{I}, \psi_{\omega l}^{I I}$ and $\psi_{\omega l}^{I I I}$ to be the same. In region I, we have [using the asymptotic form given in Eq. 4.4 ] ]

$$
W=2 i \operatorname{Im}\left(\psi_{\omega l, r_{*}}^{I} \psi_{\omega l}^{I *}\right)=-2 i \omega .
$$

In region II, we can use the general form given in Eq. [5.12] [or the explicit form of Eq. [5.13] ] and get

$$
W=2 i \operatorname{Im}\left(\psi_{\omega l, r_{*}}^{I I} \psi_{\omega l}^{I I *}\right)=2 i\left(\left|a_{+}\right|^{2}-\left|a_{-}\right|^{2}\right) .
$$

When substituting $a_{+}$and $a_{-}$from Eqs. (5.14) and (5.15), we indeed obtain the same result as in region I.

Similarly, in region III we can easily calculate the Wronskian using the asymptotic form given in Eq. (5.23). We find

$$
W=2 i \operatorname{Im}\left(\psi_{\omega l, r_{*}}^{I I I} \psi_{\omega l}^{I I I *}\right)=-2 i \omega\left(|T|^{2}-|R|^{2}\right),
$$

and again after the substitution of $T$ and $R$ from Eqs. (5.21) and (5.22), we get the same result as in regions I and II.

\section{ASYMPTOTIC BEHAVIOR OF $\left\langle\hat{\Phi}^{2}\right\rangle_{r e n}$ AND $\left\langle\hat{T}_{\mu \nu}\right\rangle_{r e n}$ NEAR THE INNER HORIZON}

Now we have all the ingredients that we need in order to calculate the leading asymptotic behavior of $\left\langle\hat{\Phi}^{2}\right\rangle_{\text {ren }}$ and $\left\langle\hat{T}_{\mu \nu}\right\rangle_{r e n}$ near the inner horizon. We first start from analyzing $\left\langle\hat{\Phi}^{2}\right\rangle_{r e n}$ and then move to $\left\langle\hat{T}_{\mu \nu}\right\rangle_{r e n}$.

\section{A. $\left\langle\hat{\Phi}^{2}\right\rangle_{\text {ren }}$}

Let us begin by considering the Hartle-Hawking state. In order to find $\left\langle\hat{\Phi}^{2}\right\rangle_{H, r e n}$, we use the renormalization method described in subSec. (IIB), see Eq. 2.16. For that, we first need to find $G_{H}^{(1)}\left(x, x^{\prime}\right)^{5}$, conveniently expressed in Eq. 4.2 in terms of the inner Eddington-Finkelstein modes $f_{\omega l m}^{\mathrm{L}}(x)$ and $f_{\omega l m}^{\mathrm{R}}(x)$. As mentioned in subSec. (IIB, we take the separation between the two points $x$ and $x^{\prime}$ to be in the $t$ direction. Specifically, we choose $x=(t, r, \theta, \varphi)$ and $x^{\prime}=(t+\varepsilon, r, \theta, \varphi)$. Then, substituting $\psi_{\omega l}^{I I I}(r)$ given by Eq. 5.17) into Eq. 44.3), and the resulting $\tilde{f}_{\omega l}^{\mathrm{L}}(t, r)$ and $\tilde{f}_{\omega l}^{\mathrm{R}}(t, r)$ into Eq. (2.5), we obtain the inner Eddington-Finkelstein modes $f_{\omega l m}^{\mathrm{L}}(x)$ and $f_{\omega l m}^{\mathrm{R}}(x)$ near the inner horizon (i.e. in region III). Next, we substitute these modes [along with the expression for $\rho_{\omega l}^{\text {up }}$ we found in Eq. (3.17)] into Eq. 4.2 and get

$$
G_{H}^{(1)}\left(x, x^{\prime}\right) \cong \frac{8 \pi}{\kappa_{-}} \int_{0}^{\infty} d \omega \cos (\omega \varepsilon) \sum_{l, m}\left|C_{l m}\right|^{2}\left|F^{I I I}(r)\right|^{2}, r \rightarrow r_{-},
$$

where $F^{I I I}(r)$ is given by Eq. (5.18) and $C_{l m}$ by Eq. (2.6). As in Sec. (III), we take advantage of the fact that the leading contribution comes from large- $l$ values and switch the summation over $l$ with an integral. After we substitute for $C_{l m}$ using Eq. 2.6. and perform the summation over $m$, we find

$$
G_{H}^{(1)}\left(x, x^{\prime}\right) \cong \frac{2}{\pi\left(r_{+}-r_{-}\right)} \int_{0}^{\infty} d \omega \cos (\omega \varepsilon) \int_{0}^{\infty}\left|F^{I I I}(r)\right|^{2} \tilde{l} d \tilde{l}, \quad r \rightarrow r_{-} .
$$

\footnotetext{
${ }^{5}$ Instead of the two-point function $<\hat{\Phi}(x) \hat{\Phi}\left(x^{\prime}\right)>$ that appears in Eq. 2.16, we here use the Hadamard function $G_{H H}^{(1)}\left(x, x^{\prime}\right)$. Therefore, we need to include an extra factor of $1 / 2$ in front of $G_{H H}^{(1)}\left(x, x^{\prime}\right)$ in Eq. 2.16).
} 
The integral over $\tilde{l}$ in this expression is divergent. In order to find its correct value, we proceed as follows. We start by writing it in the form

$$
\begin{gathered}
I_{0} \equiv \int_{0}^{\infty}\left|F^{I I I}(r)\right|^{2} \tilde{l} d \tilde{l}=\pi^{-2} \int_{0}^{\infty} K_{i \omega / \kappa_{-}}\left[-2 i \tilde{l}\left(\frac{r-r_{-}}{r_{+}-r_{-}}\right)^{1 / 2}\right] K_{i \omega / \kappa_{-}}\left[2 i \tilde{l}\left(\frac{r-r_{-}}{r_{+}-r_{-}}\right)^{1 / 2}\right] \tilde{l} d \tilde{l} \\
\equiv \pi^{-2} \int_{0}^{\infty} K_{\nu}(-\mu \tilde{l}) K_{\nu}(\mu \tilde{l}) \tilde{l} d \tilde{l}
\end{gathered}
$$

where we used the fact that $\left[K_{i c}(i d)\right]^{*}=K_{i c}(-i d)$ for $c, d \in \mathbb{R}$, and defined

$$
\mu \equiv 2 i\left(\frac{r-r_{-}}{r_{+}-r_{-}}\right)^{1 / 2}, \quad \nu \equiv i \omega / \kappa_{-} .
$$

Next, we regulate this integral by adding a small, real and positive $\delta$ to the arguments of the Bessel functions, and get

$$
I_{0}=\pi^{-2} \int_{0}^{\infty} K_{\nu}(-\mu \tilde{l}) K_{\nu}(\mu \tilde{l}) \tilde{l} d \tilde{l} \rightarrow I_{\delta} \equiv \pi^{-2} \int_{0}^{\infty} K_{\nu}[(-\mu+\delta) \tilde{l}] K_{\nu}[(\mu+\delta) \tilde{l}] \tilde{l} d \tilde{l}
$$

We can now use the formula

$$
\int_{0}^{\infty} K_{\nu}(a x) K_{\nu}(b x) x d x=\frac{\pi(a b)^{-\nu}\left(a^{2 \nu}-b^{2 \nu}\right)}{2 \sin (\pi \nu)\left(a^{2}-b^{2}\right)}
$$

valid for

$$
|\operatorname{Re}(\nu)|<1, \quad \operatorname{Re}(a+b)>0
$$

and obtain

$$
I_{\delta}=\frac{\left(-\mu^{2}+\delta^{2}\right)^{-\nu}\left[(-\mu+\delta)^{2 \nu}-(\mu+\delta)^{2 \nu}\right]}{2 \pi \sin (\pi \nu)\left[(-\mu+\delta)^{2}-(\mu+\delta)^{2}\right]} .
$$

Expanding $I_{\delta}$ in powers of $\delta$ and substituting the expressions for $\mu$ and $\nu$, we have

$$
I_{\delta}=\frac{1}{8 \pi \delta}\left(\frac{r-r_{-}}{r_{+}-r_{-}}\right)^{-1 / 2}-\frac{\omega}{8 \pi \kappa_{-}}\left(\frac{r-r_{-}}{r_{+}-r_{-}}\right)^{-1} \operatorname{coth}\left(\frac{\pi \omega}{\kappa_{-}}\right)+\mathcal{O}(\delta) .
$$

We see that the divergent $(\operatorname{as} \delta \rightarrow 0)$ term in $I_{\delta}$ is independent of $\omega$ and thus does not contribute to $G_{H}^{(1)}\left(x, x^{\prime}\right)$ when substituted in Eq. 6.1) (this term is a "blind spot" in the language of [33]). As a result, we can remove this term and then take the limit $\delta \rightarrow 0$. The resulting regularized integral over $\tilde{l}$ is

$$
\left[I_{0}\right]_{r e g}=-\frac{\omega}{8 \pi \kappa_{-}}\left(\frac{r-r_{-}}{r_{+}-r_{-}}\right)^{-1} \operatorname{coth}\left(\frac{\pi \omega}{\kappa_{-}}\right)
$$

and after substituting it to Eq. 6.1, we get

$$
G_{H}^{(1)}\left(x, x^{\prime}\right) \cong \frac{2}{\pi\left(r_{+}-r_{-}\right)} \int_{0}^{\infty} d \omega \cos (\omega \varepsilon)\left[I_{0}\right]_{r e g}=-\frac{1}{4 \pi^{2} \kappa_{-}\left(r-r_{-}\right)} \int_{0}^{\infty} \omega \operatorname{coth}\left(\frac{\pi \omega}{\kappa_{-}}\right) \cos (\omega \varepsilon) d \omega, r \rightarrow r_{-} .
$$


We can now move on to consider the second term in the right hand side of Eq. (2.16), the DeWitt-Schwinger counterterm $G_{D S}\left(x, x^{\prime}\right)$. We have a massless $(m=0)$, minimally-coupled $(\xi=0)$ scalar field in Reissner-Nordström spacetime $(R=0)$. Therefore, substituting $m=\xi=R=0$ in Eq. (2.17), we get

$$
G_{D S}\left(x, x^{\prime}\right)=\frac{1}{8 \pi^{2} \sigma}+\frac{1}{96 \pi^{2}} R_{\mu \nu} \frac{\sigma^{; \mu} \sigma^{; \nu}}{\sigma} .
$$

Now, recall that $\sigma$ is the biscalar of geodetic separation and that we took the points $x$ and $x^{\prime}$ to be separated along the $t$ direction: $x=(t, r, \theta, \varphi)$ and $x^{\prime}=(t+\varepsilon, r, \theta, \varphi)$. Then, we can look at the (shortest) geodesic that connects $x$ and $x^{\prime}$ and expand it in powers of $\varepsilon$. For a general metric function $f(r)$ [i.e. $\left(1-2 M / r+Q^{2} / r^{2}\right) \rightarrow f(r)$ ], we get the following expansion of $G_{D S}\left(x, x^{\prime}\right)$ in powers of $\varepsilon$ :

$$
G_{D S}\left(x, x^{\prime}\right)=-\frac{1}{4 \pi^{2} f(r)} \varepsilon^{-2}+\frac{f^{\prime}(r)^{2}}{192 \pi^{2} f(r)}-\frac{1}{48 \pi^{2} f(r)} R_{00}+\mathcal{O}\left(\varepsilon^{2}\right),
$$

and for the Reissner-Nordström metric, $f(r)=\left(1-2 M / r+Q^{2} / r^{2}\right)$, we obtain near $r=r_{-}$[henceforth, we remove the $\mathcal{O}\left(\varepsilon^{2}\right)$ terms]

$$
G_{D S}\left(x, x^{\prime}\right) \cong \frac{1}{8 \pi^{2} \kappa_{-}\left(r-r_{-}\right)} \varepsilon^{-2}-\frac{\kappa_{-}}{96 \pi^{2}\left(r-r_{-}\right)}, r \rightarrow r_{-} .
$$

Using

$$
\varepsilon^{-2}=-\int_{0}^{\infty} \omega \cos (\omega \varepsilon) d \omega
$$

we can also write

$$
G_{D S}\left(x, x^{\prime}\right) \cong-\frac{1}{8 \pi^{2} \kappa_{-}\left(r-r_{-}\right)} \int_{0}^{\infty} \omega \cos (\omega \varepsilon) d \omega-\frac{\kappa_{-}}{96 \pi^{2}\left(r-r_{-}\right)}, r \rightarrow r_{-} .
$$

Now, we can finally find the leading behavior of $\left\langle\hat{\Phi}^{2}\right\rangle_{H, r e n}$ near $r=r_{-}$. Substituting Eqs. (6.2) and $(6.3)$ into (2.16), we get

$$
\begin{aligned}
& \left\langle\hat{\Phi}^{2}(x)\right\rangle_{H, r e n}=\lim _{x^{\prime} \rightarrow x}\left[\left\langle\hat{\Phi}(x) \hat{\Phi}\left(x^{\prime}\right)\right\rangle_{H}-G_{D S}\left(x, x^{\prime}\right)\right]=\lim _{x^{\prime} \rightarrow x}\left[\frac{1}{2} G_{H}^{(1)}\left(x, x^{\prime}\right)-G_{D S}\left(x, x^{\prime}\right)\right] \\
& \cong-\frac{1}{8 \pi^{2} \kappa_{-}\left(r-r_{-}\right)} \int_{0}^{\infty} \omega\left[\operatorname{coth}\left(\frac{\pi \omega}{\kappa_{-}}\right)-1\right] d \omega+\frac{\kappa_{-}}{96 \pi^{2}\left(r-r_{-}\right)}=0, \quad r \rightarrow r_{-} .
\end{aligned}
$$

Note that in the second line the limit $\varepsilon \rightarrow 0$ was taken before the integration was carried out. Of course, we could have performed the integration first (using the Abel-summation integral, see [30]) and only then take the $\varepsilon \rightarrow 0$ limit and get the same result. We obtained that the coefficient of the expected leading divergence $\left[\propto\left(r-r_{-}\right)^{-1}\right]$ of $\left\langle\hat{\Phi}^{2}\right\rangle_{H, r e n}$ near the inner horizon vanishes. As a result, according to this analysis $\left\langle\hat{\Phi}^{2}\right\rangle_{H, \text { ren }}$ may have a weaker divergence, such as $\propto \log \left(r-r_{-}\right)$, or it may be regular. This is consistent with new numerical results [18] showing that $\left\langle\hat{\Phi}^{2}\right\rangle_{H, r e n}$ approaches a finite value at the inner horizon and is therefore regular.

As for the Unruh state, we obtain from subtracting Eqs. 4.2) and 4.1] that

$$
G_{H}^{(1)}\left(x, x^{\prime}\right)-G_{U}^{(1)}\left(x, x^{\prime}\right)=\int_{0}^{\infty} d \omega \sum_{l, m}\left|\tau_{\omega l}^{\mathrm{up}}\right|^{2}\left[\operatorname{coth}\left(\frac{\pi \omega}{\kappa_{+}}\right)-1\right]\left\{f_{\omega l m}^{\mathrm{R}}(x), f_{\omega l m}^{\mathrm{R} *}\left(x^{\prime}\right)\right\} .
$$

Then, from Eq. 2.16) we get

$$
\left\langle\hat{\Phi}^{2}(x)\right\rangle_{H, r e n}-\left\langle\hat{\Phi}^{2}(x)\right\rangle_{U, r e n}=\int_{0}^{\infty} d \omega \sum_{l, m}\left|\tau_{\omega l}^{\mathrm{up}}\right|^{2}\left[\operatorname{coth}\left(\frac{\pi \omega}{\kappa_{+}}\right)-1\right]\left|f_{\omega l m}^{\mathrm{R}}(x)\right|^{2} .
$$

This quantity does not contribute at the leading order, since its dominant part comes from small $\omega$ and large $l$ values, and is highly suppressed by $\tau_{\omega l}^{\mathrm{up}}$ in this domain. Therefore, the leading divergence of $\left\langle\hat{\Phi}^{2}\right\rangle_{U, r e n}$ will be the same as that of $\left\langle\hat{\Phi}^{2}\right\rangle_{H, r e n}$, which is vanishing. This again matches the numerical results [18]. 
B. $\left\langle\hat{T}_{\mu \nu}\right\rangle_{r e n}$

The same argument that appears in the end of the previous subsection for $\hat{\Phi}^{2}$ applies to $\hat{T}_{\mu \nu}$ as well, hence the leading divergence of the stress-energy tensor should be the same for both the Unruh and Hartle-Hawking states. As in the previous subsection, we choose to calculate the expectation value in the Hartle-Hawking state (because the mode-sum expressions are less complicated). We follow the renormalization prescription mentioned in Sec. (IIB).

Instead of calculating each of the components of $\left\langle\hat{T}_{\mu \nu}\right\rangle_{H, r e n}$ independently, we can use the conservation of stressenergy and Eq. 2.20 in order to relate the various components, ending up with only one independent component. To see this, note that we found that the "leading divergence" of $\left\langle\hat{\Phi}^{2}\right\rangle_{H, r e n}$ near the inner horizon is $\propto\left(r-r_{-}\right)^{-1}$ [see, for example, Eq. [6.2 or [6.4 ] and that the corresponding coefficient vanishes. As a result, we expect the leading divergence of $\left\langle\hat{T}_{\mu \nu}\right\rangle_{H, \text { ren }}$ to be $\propto\left(r-r_{-}\right)^{-2}$ with a potentially nonvanishing coefficient [similarly to $\left\langle\hat{T}_{\mu \nu}\right\rangle_{B, \text { ren }}$ in the exterior region near $r=r_{+}$, see Eq. [3.18]]. Recalling that we have a spherical symmetry, we can therefore write

$$
\left\langle\hat{T}_{\nu}^{\mu}\right\rangle_{H, r e n} \cong\left(r-r_{-}\right)^{-2}\left(\begin{array}{cccc}
c_{t} & 0 & 0 & 0 \\
0 & c_{r} & 0 & 0 \\
0 & 0 & c_{\theta} & 0 \\
0 & 0 & 0 & c_{\theta}
\end{array}\right), r \rightarrow r_{-} .
$$

In order to use the conservation of energy and momentum, we quote the following formula:

$$
A_{\nu ; \mu}^{\mu}=\frac{1}{\sqrt{-g}}\left(\sqrt{-g} A_{\nu}^{\mu}\right)_{, \mu}-\frac{1}{2} g_{\mu \sigma, \nu} A^{\mu \sigma},
$$

valid for a general rank-2 symmetric tensor $A_{\nu}^{\mu}$. Applying it to the conservation equation

$$
\left\langle\hat{T}_{\nu ; \mu}^{\mu}\right\rangle_{H, r e n}=0
$$

and choosing $\nu=r$, we get at leading order

$$
0=\left\langle\hat{T}_{r ; \mu}^{\mu}\right\rangle_{H, r e n} \cong\left\langle\hat{T}_{r, r}^{r}\right\rangle_{H, r e n}-\frac{1}{2} g_{t t, r}\left\langle\hat{T}^{t t}\right\rangle_{H, r e n}-\frac{1}{2} g_{r r, r}\left\langle\hat{T}^{r r}\right\rangle_{H, r e n} \cong-\frac{1}{2}\left(r-r_{-}\right)^{-3}\left(c_{t}+3 c_{r}\right), r \rightarrow r_{-},
$$

hence

$$
c_{t}+3 c_{r}=0 .
$$

Next, we would like to use Eq. 2.20). Since $T_{\text {anomaly }}$ is a local, geometric quantity built from curvature scalars [see Eq. [2.19]], it is regular at the inner horizon. Moreover, since the divergence (if any) of $\left\langle\hat{\Phi}^{2}\right\rangle_{H \text {,ren }}$ at $r=r_{-}$is weaker than $\propto\left(r-r_{-}\right)^{-1}$, the divergence of $\square\left\langle\hat{\Phi}^{2}\right\rangle_{H, r e n}$ is weaker than $\propto\left(r-r_{-}\right)^{-2}{ }^{6}$. Therefore, according to Eq. 2.20, the trace of the stress-energy tensor vanishes at leading order $\left[\propto\left(r-r_{-}\right)^{-2}\right]$ and we have from Eq. 6.5):

$$
c_{t}+c_{r}+2 c_{\theta}=0
$$

Combining Eqs. 6.6 and 6.7, we obtain

$$
c_{r}=c_{\theta}=-\frac{1}{3} c_{t}
$$

As mentioned above, we see that there is only one independent component of $\left\langle\hat{T}_{\mu \nu}\right\rangle_{H, r e n}$ at leading order. We choose to calculate $\left\langle\hat{T}_{t t}\right\rangle_{H, r e n}$. Now, since the trace $\left\langle\hat{T}_{\mu}^{\mu}\right\rangle_{H, r e n}$ does not contribute at leading order, it is enough to calculate $\left\langle\left\{\hat{\Phi}_{, t}(x) \hat{\Phi}_{, t}\left(x^{\prime}\right)\right\}\right\rangle_{H}$ instead of the whole expression for $\left\langle\hat{T}_{t t}\left(x, x^{\prime}\right)\right\rangle_{H}{ }^{7}$. As before, we take the splitting to be in the $t$-direction and choose $x=(t, r, \theta, \varphi)$ and $x^{\prime}=(t+\varepsilon, r, \theta, \varphi)$. It is easy to see that the mode-sum expression for this

\footnotetext{
${ }^{6}$ For example, if $\left\langle\hat{\Phi}^{2}>_{H, r e n} \propto \log \left(r-r_{-}\right)\right.$than $\square<\hat{\Phi}^{2}>_{H, \text { ren }}$ is regular at $r=r_{-}$.

7 Notice that, as discussed in subSec. (II B 1), all the derivatives in the expression for the stress-energy tensor are taken at the same point $x$, and the bi-vector of parallel transport is absent (it appears in the counterterm instead).
} 
quantity is the same as that of $G_{H}^{(1)}\left(x, x^{\prime}\right)$, given in Eq. 6.2 , up to an extra factor of $\omega^{2}$ in the integrand. Thus, we have

$$
\left\langle\hat{T}_{t t}\left(x, x^{\prime}\right)\right\rangle_{H} \cong \frac{1}{2}\left\langle\left\{\hat{\Phi}_{, t}(x), \hat{\Phi}_{, t}\left(x^{\prime}\right)\right\}\right\rangle_{H} \cong-\frac{1}{8 \pi^{2} \kappa_{-}\left(r-r_{-}\right)} \int_{0}^{\infty} \omega^{3} \operatorname{coth}\left(\frac{\pi \omega}{\kappa_{-}}\right) \cos (\omega \varepsilon) d \omega, r \rightarrow r_{-} .
$$

In [33], the general form of the counterterm $C_{\mu \nu}^{D S}$ in terms of Christensen's original one [35] was found, and its expansion in powers of $\varepsilon$ (the $t$-splitting parameter) was explicitly obtained for a massless, minimally coupled scalar field in Schwarzschild spacetime. The extension of this result to Reissner-Nordström spacetime is given by ${ }^{8}$

$$
C_{\mu \nu}^{D S}\left(x, x^{\prime}\right)=\int_{0}^{\infty}\left(a_{\mu \nu} \omega^{3}+b_{\mu \nu} \omega+c_{\mu \nu} \ln (\omega)+d_{\mu \nu} \frac{1}{\omega+\mu e^{-\gamma}}\right) \cos (\omega \varepsilon) d \omega+e_{\mu \nu},
$$

where the coefficients $b_{t t}, c_{t t}$ and $d_{t t}$ do not contribute at leading order and

$$
\begin{gathered}
a_{t t} \cong-\frac{1}{8 \pi^{2} \kappa_{-}\left(r-r_{-}\right)}, r \rightarrow r_{-}, \\
e_{t t} \cong-\frac{\kappa_{-}^{3}}{960 \pi^{2}\left(r-r_{-}\right)}, r \rightarrow r_{-} .
\end{gathered}
$$

Substituting the expressions we obtained for the counterterm and the (split) stress-energy tensor [given by Eqs. [6.9) and 6.10] into Eq. 2.18 and taking the limit $\varepsilon \rightarrow 0$, we get

$$
\left\langle\hat{T}_{t t}\right\rangle_{H, r e n} \cong-\frac{1}{8 \pi^{2} \kappa_{-}\left(r-r_{-}\right)} \int_{0}^{\infty}\left[\operatorname{coth}\left(\frac{\pi \omega}{\kappa_{-}}\right)-1\right] \omega^{3} d \omega+\frac{\kappa_{-}^{3}}{960 \pi^{2}\left(r-r_{-}\right)}=0, r \rightarrow r_{-} .
$$

Using Eq. 6.8), we find that the coefficient of the expected leading divergence of all the components of $\left\langle\hat{T}_{\mu \nu}\right\rangle_{H, r e n}$ near the inner horizon vanishes. As mentioned above, the same applies to $\left\langle\hat{T}_{\mu \nu}\right\rangle_{U, r e n}$.

\section{DISCUSSION}

In this work we considered a massless, minimally coupled quantum scalar field on a RN black hole background, and studied the asymptotic behavior of $\left\langle\hat{\Phi}^{2}\right\rangle_{\text {ren }}$ and $\left\langle\hat{T}_{\mu \nu}\right\rangle_{\text {ren }}$ near the inner and outer horizons in quantum states in which they are expected to diverge. Our strategy was to analyze the modes of the scalar field near the horizons, where analytic expressions can be obtained. Then, using expressions for these expectation values as mode-sums of these modes, we obtained their leading asymptotic behavior near the horizons. In this calculation we made the assumption that this asymptotic behavior is determined by the large- llimit of the modes, and we found agreement with [16 18.

In section III, we used this analytical approach to obtain the known divergence of $\left\langle\hat{\Phi}^{2}\right\rangle_{\text {ren }}$ and $\left\langle\hat{T}_{\mu \nu}\right\rangle_{\text {ren }}$, evaluated in Boulware state, at the event horizon. We derived new and explicit analytic expressions for the asymptotic forms of these expectation values, which correspond to the numerical results obtained in Refs. [16, 17.

Then, in the rest of the paper, we applied our approach to the calculation of the asymptotic behavior of $\left\langle\hat{\Phi}^{2}\right\rangle_{\text {ren }}$ and $\left\langle\hat{T}_{\mu \nu}\right\rangle_{r e n}$, evaluated in Unruh and Hartle-Hawking states, near the inner (Cauchy) horizon. We found that the coefficient of this "leading" divergence vanishes, and therefore the divergence, if it occurs, is weaker than expected a priori. These a priori expectations may originate from various different directions. First, by examining the expressions for the counterterms or the mode-sums near the inner horizon [see, for example, Eq. [6.3] ], we might expect these strong divergences. For example, in the case of $\left\langle\hat{\Phi}^{2}\right\rangle_{r e n}$, this yields a $\propto\left(r-r_{-}\right)^{-1}$ divergence. Second, these strong divergences exactly correspond to the ones found in section III in the case of the Boulware state and the outer (event) horizon. One may expect that this is a general behavior of this kinds of expectation values near horizons. Finally, the same strong divergences were obtained in Ref. [19] for a conformally (rather than minimally) coupled scalar field,

\footnotetext{
${ }^{8}$ I thank A. Levi for providing this counterterm for me.
} 
but under a certain approximation that was carried out. Our goal was thus to calculate the leading asymptotic forms of $\left\langle\hat{\Phi}^{2}\right\rangle_{\text {ren }}$ and $\left\langle\hat{T}_{\mu \nu}\right\rangle_{\text {ren }}$ near the inner horizon without recourse to the approximation methods used before and for a minimally-coupled scalar field.

In the case of the Unruh state (describing an evaporating BH), it was shown in Ref. 13] that the RSET has to diverge on at least one of the inner horizons (in the RN case). Therefore, our findings show that this divergence is weaker than might be expected a priori. This, in turn, opens the door for a scenario in which the resulting modification of the metric is finite.

In order to obtain the exact asymptotic form near the horizon (and not only the leading-order one), numerical calculation and mode-sum of the modes should be employed. This study will be published in a subsequent paper and its results match the ones obtained in this note [18].

It will be interesting to apply our analytical approach to the calculation of the asymptotic form of the RSET of a conformally coupled scalar field, and test whether the approximate results of [19] are valid near the inner horizon. Furthermore, an extension to a more realistic model, such as to a Kerr black hole background or a quantum electromagnetic field instead of a scalar one, will add an important contribution to the picture.

\section{ACKNOWLEDGMENTS}

I would like to thank Amos Ori for his guidance throughout the execution of this work. This research was supported by the Israel Science Foundation under Grant No. 1696/15 and by the I-CORE Program of the Planning and Budgeting Committee.

\section{APPENDIX: THE RADIAL FUNCTION IN THE INTERNAL REGION OF THE BLACK HOLE}

In this appendix we derive the expressions (5.14), (5.15), (5.19) and 5.20 for the coefficients in the definitions of $\psi_{\omega l}^{I I}$ and $\psi_{\omega l}^{I I I}$. The strategy is to take the $r_{*} \rightarrow \pm \infty$ limits of $\psi_{\omega l}^{I I}$ and compare it with the limits of $\psi_{\omega l}^{I}$ and $\psi_{\omega l}^{I I}$ corresponding to the overlapping regions with $\psi_{\omega l}^{I I}$. We begin with the expression for $\psi_{\omega l}^{I I}$ given in Eq. (5.13), which we reproduce here for convenience,

$$
\begin{gathered}
\psi_{\omega l}^{I I}=\frac{r}{\sqrt{l}}\left[\left(r_{+}-r\right)\left(r-r_{-}\right)\right]^{-1 / 4}\left\{a_{+} \exp \left[-i \tilde{l} \arctan \left(\frac{r-M}{\left[\left(r_{+}-r\right)\left(r-r_{-}\right)\right]^{1 / 2}}\right)\right]\right. \\
\left.+a_{-} \exp \left[i \tilde{l} \arctan \left(\frac{r-M}{\left[\left(r_{+}-r\right)\left(r-r_{-}\right)\right]^{1 / 2}}\right)\right]\right\} .
\end{gathered}
$$

In order to obtain $\psi_{\omega l}^{I I}$ at the limits $r \rightarrow r_{ \pm}$, we first consider the exponents at these limits,

$$
\arctan \left(\frac{r-M}{\sqrt{\left(r_{+}-r\right)\left(r-r_{-}\right)}}\right)=\left\{\begin{array}{c}
\frac{\pi}{2}-2\left(\frac{r_{+}-r}{r_{+}-r_{-}}\right)^{1 / 2}+\mathcal{O}\left[\left(r_{+}-r\right)^{3 / 2}\right], r \rightarrow r_{+} \\
-\frac{\pi}{2}+2\left(\frac{r-r_{-}}{r_{+}-r_{-}}\right)^{1 / 2}+\mathcal{O}\left[\left(r-r_{-}\right)^{3 / 2}\right], r \rightarrow r_{-}
\end{array}\right.
$$

from which we get

$$
\begin{gathered}
\psi_{\omega l}^{I I} \cong \frac{r_{+}}{\sqrt{\tilde{l}}}\left[\left(r_{+}-r\right)\left(r_{+}-r_{-}\right)\right]^{-1 / 4}\left\{a_{+}(-i)^{\tilde{l}} \exp \left[2 i \tilde{l}\left(\frac{r_{+}-r}{r_{+}-r_{-}}\right)^{1 / 2}\right]+\right. \\
\left.+a_{-} i^{\tilde{l}} \exp \left[-2 i \tilde{l}\left(\frac{r_{+}-r}{r_{+}-r_{-}}\right)^{1 / 2}\right]\right\}, r \rightarrow r_{+}
\end{gathered}
$$

and

$$
\psi_{\omega l}^{I I} \cong \frac{r_{-}}{\sqrt{\tilde{l}}}\left[\left(r_{+}-r_{-}\right)\left(r-r_{-}\right)\right]^{-1 / 4}\left\{a_{+} i^{\tilde{l}} \exp \left[-2 i \tilde{l}\left(\frac{r-r_{-}}{r_{+}-r_{-}}\right)^{1 / 2}\right]+\right.
$$




$$
\left.+a_{-}(-i)^{\tilde{l}} \exp \left[2 i \tilde{l}\left(\frac{r-r_{-}}{r_{+}-r_{-}}\right)^{1 / 2}\right]\right\}, r \rightarrow r_{-} .
$$

Next, to get $\psi_{\omega l}^{I}$ and $\psi_{\omega l}^{I I I}$ in the overlapping regions, we take the $r \rightarrow-\infty$ limit of $\psi_{\omega l}^{I}$ and the $r \rightarrow \infty$ limit of $\psi_{\omega l}^{I I I}$. Using the following asymptotic form of the Bessel $\mathrm{K}$ function,

$$
K_{c}(y) \cong \sqrt{\frac{\pi}{2 y}} \exp (-y), y \rightarrow \infty
$$

valid for any complex number $c$, we can find the desired asymptotic forms of $F^{I}(r)$ and $F^{I I I}(r)$ given in Eqs. 5.5 and 5.18,

$$
\begin{aligned}
& F^{I}(r) \cong \frac{1}{2 \sqrt{\pi \tilde{l}}}\left(\frac{r_{+}-r}{r_{+}-r_{-}}\right)^{-1 / 4} \exp \left[2 i \tilde{l}\left(\frac{r_{+}-r}{r_{+}-r_{-}}\right)^{1 / 2}\right], r \rightarrow-\infty, \\
& F^{I I I}(r) \cong \frac{1}{2 \sqrt{\pi \tilde{l}}}\left(\frac{r-r_{-}}{r_{+}-r_{-}}\right)^{-1 / 4} \exp \left[2 i \tilde{l}\left(\frac{r-r_{-}}{r_{+}-r_{-}}\right)^{1 / 2}\right], r \rightarrow \infty .
\end{aligned}
$$

Substituting these expressions in 5.4 and (5.17), we obtain

$$
\psi_{\omega l}^{I}(r) \cong \frac{1}{2 \sqrt{\pi \tilde{l}}}\left(\frac{r_{+}-r}{r_{+}-r_{-}}\right)^{-1 / 4}\left\{p_{1}^{I} \exp \left[2 i \tilde{l}\left(\frac{r_{+}-r}{r_{+}-r_{-}}\right)^{1 / 2}\right]+p_{2}^{I} \exp \left[-2 i \tilde{l}\left(\frac{r_{+}-r}{r_{+}-r_{-}}\right)^{1 / 2}\right]\right\}, r \rightarrow-\infty
$$

and

$$
\psi_{\omega l}^{I I I} \cong \frac{1}{2 \sqrt{\pi \tilde{l}}}\left(\frac{r-r_{-}}{r_{+}-r_{-}}\right)^{-1 / 4}\left\{p_{1}^{I I I} \exp \left[2 i \tilde{l}\left(\frac{r-r_{-}}{r_{+}-r_{-}}\right)^{1 / 2}\right]+p_{2}^{I I I} \exp \left[-2 i \tilde{l}\left(\frac{r-r_{-}}{r_{+}-r_{-}}\right)^{1 / 2}\right]\right\}, r \rightarrow \infty .
$$

We can now compare 7.1 with $(7.3)$, and $(7.2$ with 7.4 . We find

$$
\begin{gathered}
a_{+}=\left(\frac{\kappa_{+}}{2 \pi}\right)^{1 / 2} i^{\tilde{l}} p_{1}^{I}=\left(\frac{\kappa_{+}}{2 \pi}\right)^{1 / 2} i^{\tilde{l}-1} e^{i \frac{\pi}{4}} \tilde{l}^{i \omega / \kappa_{+}} e^{-i \omega r_{+}} \Gamma\left(1-i \omega / \kappa_{+}\right) e^{-\frac{\pi \omega}{2 \kappa_{+}}}, \\
a_{-}=\left(\frac{\kappa_{+}}{2 \pi}\right)^{1 / 2}(-i)^{\tilde{l}} p_{2}^{I}=\left(\frac{\kappa_{+}}{2 \pi}\right)^{1 / 2}(-i)^{\tilde{l}} e^{i \frac{\pi}{4}} \tilde{l}{ }^{i \omega / \kappa_{+}} e^{-i \omega r_{+}} \Gamma\left(1-i \omega / \kappa_{+}\right) e^{\frac{\pi \omega}{2 \kappa_{+}}}, \\
p_{1}^{I I I}=\left(\frac{\kappa_{-}}{2 \pi}\right)^{-1 / 2}(-i)^{\tilde{l}} a_{-}=\frac{r_{-}}{r_{+}}(-1)^{\tilde{l}} e^{-i \frac{3 \pi}{4}} \tilde{l}^{i \omega / \kappa_{+}} e^{-i \omega r_{+}} \Gamma\left(1-i \omega / \kappa_{+}\right) e^{\frac{\pi \omega}{2 \kappa_{+}}}, \\
p_{2}^{I I I}=\left(\frac{\kappa_{-}}{2 \pi}\right)^{-1 / 2} i^{\tilde{l}} a_{+}=\frac{r_{-}}{r_{+}}(-1)^{\tilde{l}} e^{-i \frac{\pi}{4}} \tilde{l} \tilde{l}^{i \omega / \kappa_{+}} e^{-i \omega r_{+}} \Gamma\left(1-i \omega / \kappa_{+}\right) e^{-\frac{\pi \omega}{2 \kappa_{+}}}
\end{gathered}
$$

which are the expressions given in Eqs. 5.14, 5.15, 5.19 and 5.20.

[1] M. L. Gnedin and N. Y. Gnedin, Destruction of the Cauchy horizon in the Reissner-Nordström black hole, Class. Quantum Grav. 10, 1083 (1993).

[2] P. R. Brady and J. D. Smith, Black hole singularities: A Numerical approach, Phys. Rev. Lett. 75, 1256 (1995).

[3] L. M. Burko, Structure of the black hole's Cauchy horizon singularity, Phys. Rev. Lett. 79, 4958 (1997). 
[4] A. Ori, Structure of the singularity inside a realistic rotating black hole, Phys. Rev. Lett. 68, 2117 (1992).

[5] A. Ori, Evolution of linear gravitational and electromagnetic perturbations inside a Kerr black hole, Phys. Rev. D 61, 024001 (2000).

[6] A. Ori, Oscillatory null singularity inside realistic spinning black holes, Phys. Rev. Lett. 83, 5423 (1999).

[7] P. R. Brady, S. Droz, and S. M. Morsink, The Late time singularity inside nonspherical black holes, Phys. Rev. D 58, 084034 (1998).

[8] D. Marolf and A. Ori, Outgoing gravitational shock wave at the inner horizon: The late-time limit of black hole interiors, Phys. Rev. D 86, 124026 (2012).

[9] E. Eilon and A. Ori, Numerical study of the gravitational shock wave inside a spherical charged black hole, Phys. Rev. D 94, 104060 (2016).

[10] S. W. Hawking, Black hole explosions?, Nature 248, 30 (1974).

[11] S. W. Hawking, Particle creation by black holes, Comm. Math. Phys. 43, 199 (1975).

[12] N. D. Birrell and P. C. W. Davies, On falling through a black hole into another universe, Nature 272, 35 (1978).

[13] W.A. Hiscock, Quantum-mechanical instability of the Kerr-Newman black-hole interior, Phys. Rev. D 21, 2057 (1980).

[14] A. C. Ottewill and E. Winstanley, Renormalized stress tensor in Kerr space-time: General results, Phys. Rev. D 62, 084018 (2000).

[15] A. Ori, Inside an evaporating two-dimensional charged black hole, arXiv:gr-qc/0609092 (2006).

[16] P. R. Anderson, W. A. Hiscock, and D. A. Samuel, Stress-energy tensor of quantized scalar fields in static spherically symmetric spacetimes, Phys. Rev. D 51, 4337 (1995).

[17] P. R. Anderson, A method to compute $\left\langle\phi^{2}\right\rangle$ in asymptotically flat, static, spherically symmetric spacetimes, Phys. Rev. D 41, 1152 (1990).

[18] A. Lanir, A. Ori, and O. Sela (to be published).

[19] C. G. Huang, Thermal stress-energy tensor of a scalar field in Reissner-Nordström space-time, Phys. Lett. A 164, 384 (1992).

[20] T. Zannias, Renormalized thermal stress tensor for arbitrary static space-times, Phys. Rev. D 30, 1161 (1984).

[21] V. P. Frolov and A. I. Zel'nikov, Killing approximation for vacuum and thermal stress-energy tensor in static space-times, Phys. Rev. D 35, 3031 (1987).

[22] P. B. Groves, P. R. Anderson, and E. D. Carlson, Method to compute the stress-energy tensor for the massless spin $\frac{1}{2}$ field in a general static spherically symmetric spacetime, Phys. Rev. D 66, 124017 (2002). 
[23] P. R. Anderson, E. Mottola, and R. Vaulin, Stress tensor from the trace anomaly in Reissner-Nordström spacetimes, Phys. Rev. D 76, 124028 (2007).

[24] M. R. Brown and A. C. Ottewill, Photon propagators and the definition and approximation of renormalized stress tensors in curved space-time, Phys. Rev. D 34, 1776 (1986).

[25] A. Lanir, A. Levi, A. Ori, and O. Sela, Two-point function of a quantum scalar field in the interior region of a ReissnerNordström black hole, Phys. Rev. D 97, 024033 (2018).

[26] D. G. Boulware, Quantum field theory in Schwarzschild and Rindler spaces, Phys. Rev. D 11, 1404 (1975).

[27] P. Candelas, Vacuum polarization in Schwarzschild spacetime, Phys. Rev. D 21, 2185 (1980).

[28] W. G. Unruh, Notes on black-hole evaporation, Phys. Rev. D 14, 870 (1976).

[29] J. B. Hartle and S. W. Hawking, Path-integral derivation of black-hole radiance, Phys. Rev. D 13, 2188 (1976).

[30] A. Levi and A. Ori, Pragmatic mode-sum regularization method for semiclassical black-hole spacetimes, Phys. Rev. D 91, 104028 (2015).

[31] A. Levi and A. Ori, Mode-sum regularization of $\left\langle\phi^{2}\right\rangle$ in the angular-splitting method, Phys. Rev. D 94, 044054 (2016).

[32] A. Levi and A. Ori, Versatile Method for Renormalized Stress-Energy Computation in Black-Hole Spacetimes, Phys. Rev. Lett. 117, 231101 (2016).

[33] A. Levi, Renormalized stress-energy tensor for stationary black holes, Phys. Rev. D 95, 025007 (2017).

[34] S. M. Christensen, Vacuum expectation value of the stress tensor in an arbitrary curved background: The covariant pointseparation method, Phys. Rev. D 14, 2490 (1976).

[35] S. M. Christensen, Regularization, renormalization, and covariant geodesic point separation, Phys. Rev. D 17, 946 (1978).

[36] B. S. DeWitt, Dynamical Theory of Groups and Fields (Gordon and Breach, New York, 1965).

[37] J. Schwinger, On Gauge Invariance and Vacuuwn Polarization, Phys. Rev. 82, 664 (1951).

[38] N. D. Birrell and P. C. W. Davies, Quantum fields in curved space (Cambridge: Cambridge University Press, 1982).

[39] S. M. Christensen and S. A. Fulling, Trace anomalies and the Hawking effect, Phys. Rev. D 15, 2088 (1977). 